\title{
The transcription factor FoxH1 (FAST) mediates Nodal signaling during anterior-posterior patterning and node formation in the mouse
}

\author{
Masamichi Yamamoto, Chikara Meno, Yasuo Sakai, Hidetaka Shiratori, Kyoko Mochida, \\ Yayoi Ikawa, Yukio Saijoh, and Hiroshi Hamada ${ }^{1}$ \\ Division of Molecular Biology, Institute for Molecular and Cellular Biology, Osaka University; CREST, Japan Science \\ and Technology Corporation, Osaka 565-0871, Japan
}

FoxH1 (FAST) is a transcription factor that mediates signaling by transforming growth factor- $\beta$, Activin, and Nodal. The role of FoxH1 in development has now been investigated by the generation and analysis of FoxH1-deficient $\left(\mathrm{FoxH1}^{-/-}\right)$mice. The FoxH1 ${ }^{-/-}$embryos showed various patterning defects that recapitulate most of the defects induced by the loss of Nodal signaling. A substantial proportion of FoxH1 ${ }^{-/-}$embryos failed to orient the anterior-posterior (A-P) axis correctly, as do mice lacking Cripto, a coreceptor for Nodal. In less severely affected FoxH1-/- embryos, A-P polarity was established, but the primitive streak failed to elongate, resulting in the lack of a definitive node and its derivatives. Heterozygosity for nodal renders the FoxH1 ${ }^{-/-}$phenotype more severe, indicative of a genetic interaction between FoxH1 and nodal. The expression of FoxH1 in the primitive endoderm rescued the A-P patterning defects, but not the midline defects, of Fox H1 $1^{-1-}$ mice. These results indicate that a Nodal-FoxH1 signaling pathway plays a central role in A-P patterning and node formation in the mouse.

[Key Words: anterior-posterior patterning; FoxH1; gastrulation; Nodal; node]

Received January 30, 2001; revised version accepted March 28, 2001.

Factors related to transforming growth factor- $\beta$ (TGF- $\beta$ ) control multiple aspects of early development in vertebrates. One such factor, Nodal (Zhou et al. 1993), is a potent signaling molecule that is required for specification of the anterior-posterior (A-P) axis, formation of the primitive streak, and left-right patterning (Schier and Shen 2000). In general, TGF- $\beta$-related factors initiate intracellular signaling by interacting with type I and type II receptors on the cell surface, which in turn activate intracellular effectors known as Smad proteins (Massague 1998). The activated Smad proteins then translocate to the nucleus, where they interact with transcription factors and thereby regulate the expression of target genes. Although the Nodal signaling pathway remains to be fully characterized, genetic evidence suggests that ALK4 (ActRIB) functions as a type I receptor and that ActRIIA and ActRIIB serve as type II receptors in this pathway. Nodal activity is modulated by extracellular cofactors that belong to the EGF-CFC family of proteins (Gritsman et al. 1999), as well as by inhibitors that belong to the

${ }^{1}$ Corresponding author.

E-MAIL hamada@imcb.osaka-u.ac.jp; FAX 81-6-6878-9846

Article and publication are at http://www.genesdev.org/cgi/doi/10.1101/ gad. 883901 .
Lefty (Meno et al. 1999) and Cerberus families (Piccolo et al. 1999). Intracellular effectors of Nodal signaling most likely include Smad2, Smad3, and Smad4; consistent with this notion, Smad2 mutant mice exhibit early patterning defects that can be explained by a lack of Nodal signaling in the extraembryonic endoderm (Nomura and Li 1998; Waldrip et al. 1998; Heyer et al. 1999).

The transcription factor FoxH1 (FAST) also mediates Nodal signaling. This protein is a winged-helix transcription factor that was initially identified in Xenopus as a transducer of Activin signaling (Chen et al. 1996). Thus, FAST-1 (the FoxH1 ortholog in Xenopus) forms a complex with Smad2 and Smad4 in response to Activin and activates a set of genes that includes Mix1 and goosecoid (Chen et al. 1997; Watanabe and Whitman 1999). However, recent studies have revealed that, in cultured mammalian cells or frog animal caps, this transcription factor also mediates signaling by TGF- $\beta$ (Labbe et al. 1998; Zhou et al. 1998; Liu et al. 1999) and Nodal (Saijoh et al. 2000). In particular, FoxH1 appears to induce asymmetric expression of lefty2 in response to Nodal signaling (Osada et al. 2000; Saijoh et al. 2000). In the mouse, FoxH1 is expressed in early embryos (until the early somite stage) but is rapidly down-regulated as nodal expression disappears (Weisberg et al. 1998; Saijoh et al. 
2000). The expression patterns of nodal and FoxH1 thus appear to overlap with each other. Together, these observations have implicated FoxH1 in Nodal signaling.

Both Smad2 and Smad3 interact with a large number of transcription factors, including TCF, NF-кB, Mix, and Gli (Whitman 1998). It has therefore remained possible that Nodal signaling is mediated in vivo by the interaction of Smad proteins with transcription factors other than FoxH1. Different transcription factors also may mediate Nodal signaling in different cell types. To determine the role of FoxH1 in Nodal signaling, we have therefore generated and characterized mutant mice that lack this transcription factor. The mutant embryos were shown to die early during embryonic development and to show various patterning defects that can be explained by a deficiency in Nodal signaling. Our results indicate that FoxH1 indeed mediates Nodal signaling during A-P patterning and node formation.

\section{Results}

\section{Embryonic mortality of FoxH1 ${ }^{-/-}$mice}

To investigate the role of FoxH1 in development, we generated mutant mice that lack this transcription factor. The FoxH1 gene is closely linked to KIF-C2 in the reverse orientation; indeed, these two genes share a common 3' untranslated region (Liu et al. 1999). We therefore determined to remove exon 1 of FoxH1, leaving KIF-C2 intact (Fig. 1A). A targeting vector was designed to insert a Frt-flanked neo gene and a loxP site into the 5' upstream region of FoxH1 and to insert an additional loxP site into intron 1. Two embryonic stem (ES) cell lines (F94, F128) that had undergone homologous recombination were obtained (Fig. 1B), and the F94 ES cells were used to generate chimeric mice. Exon 1 of FoxH1 and the neo gene were excised by crossing the chimeric animals with transgenic mice expressing the Cre recombinase (Sakai and Miyazaki 1997), resulting in the production of Fox $\mathrm{H}^{+/-}$offspring (Fig. 1C). To generate a flox (flanked by loxP) allele (FoxH1 $\left.{ }^{\text {flox }}\right)$, we treated F128 ES cells with a Flp expression vector. One resulting clone, F128-10, from which neo had been correctly excised, was used to generate mice with the Fox $H 1^{\text {flox }}$ allele (Fig. 1C). Most of the analyses described in the present study focused on FoxH1 $1^{-/-}$mice.

Both $\mathrm{FoxH1}^{+/-}$mice and FoxH1 flox/- heterozygous mice appeared normal and fertile. Genotype analysis at weaning of progeny produced from intercrosses of Fox $\mathrm{H1}^{+/-}$heterozygotes revealed the absence of homozygous mutant animals, indicating that FoxH1 $1^{-/-}$mutants die during embryonic development. The FoxH1- allele

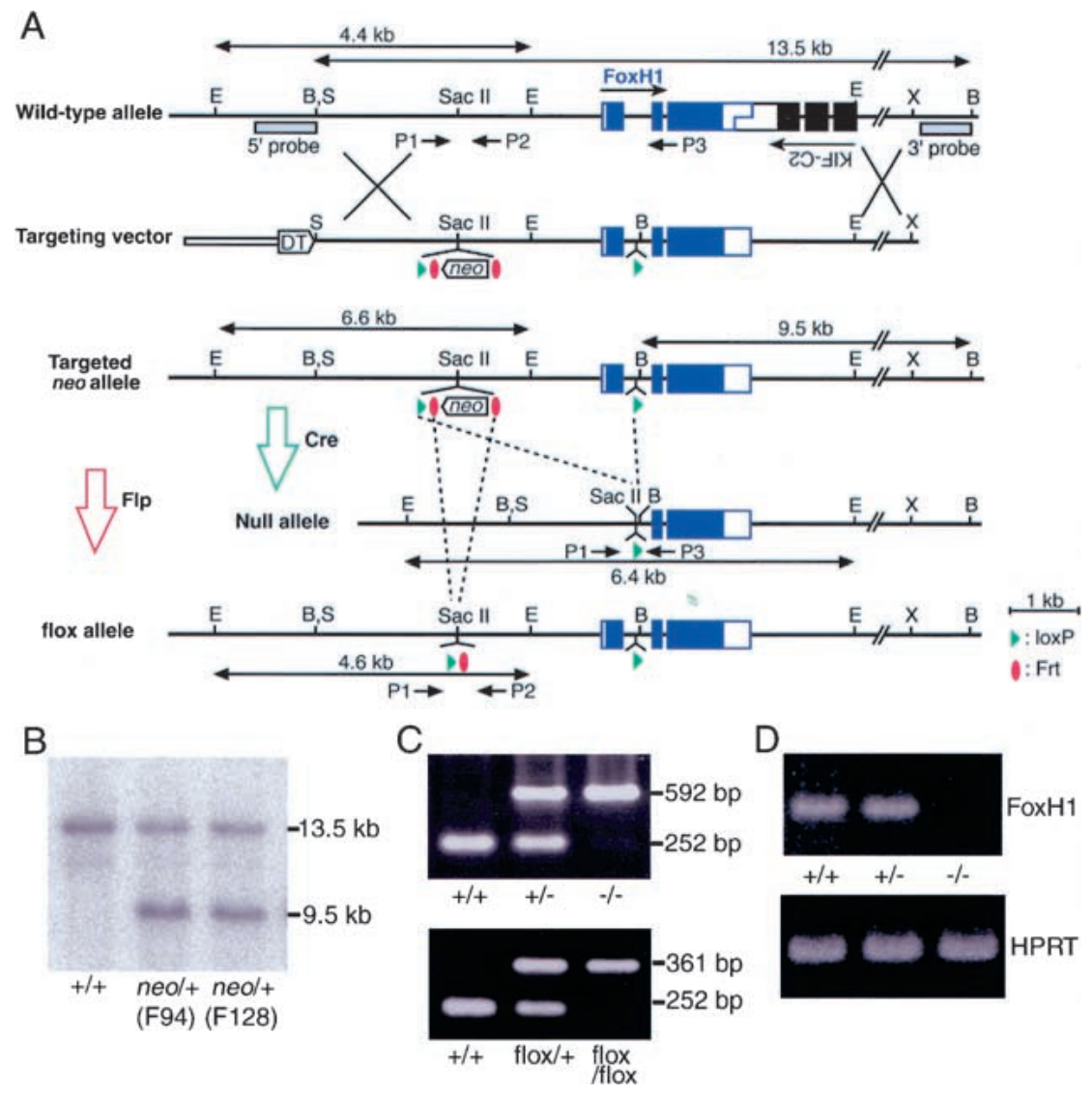

Figure 1. Generation of FoxH1 $1^{-/-}$mice. $(A)$ Targeting strategy. The genomic organization of FoxH1 is shown at the top of the panel. The KIF-C2 gene is closely linked to FoxH1 in the opposite orientation. Homologous recombination between the wild-type FoxH1 allele (exons are shown as purple solid boxes) and the targeting vector generates a neo insertional allele $\left(\right.$ Fox $\left.H 1^{\text {neo }}\right)$. A null allele $\left(\mathrm{FoxH}^{-}\right)$was created by subsequent Cre-mediated deletion of the indicated region located between loxP sites. A flox allele (FoxH $\left.1^{\text {flox }}\right)$ was generated by Flpmediated deletion of the neo cassette located between Frt sites. B indicates BamHI; E, EcoRI; S, SacI; X, XhoI; DT, diphtheria toxin resistance cassette. (B) Southern blot analysis of two correctly targeted ES cell clones (F94, F128). Genomic DNA was digested with EcoRI and subjected to hybridization with the $3^{\prime}$ probe indicated in $A$. The sizes of hybridized fragments are shown in kilobases. (C) PCR analysis of offspring obtained from intercrosses either of FoxH1 $1^{+/-}$heterozygotes (upper panel) or of FoxH $1^{\text {flox } /+}$ heterozygotes (lower panel). PCR was performed with a mixture of the three primers $(\mathrm{P} 1, \mathrm{P} 2, \mathrm{P} 3)$ shown in $A$ or with a mixture of P1 and P2 (upper and lower panels, respectively). The sizes of PCR products are shown in base pairs. $(D)$ Expression of FoxH1 and Hprt mRNA in E7.5 wild-type and FoxH1 mutant embryos was examined by RT-PCR. Note that FoxH1 mRNA was not detected in the FoxH1 $1^{-/-}$embryo. 
lacking the exon 1 is probably a null allele because FoxH1-related RNA was not detected in $\mathrm{FoxH1}^{-/-}$embryos by in situ hybridization with a full-length FoxH1 anti-sense probe (data not shown) or by reverse transcription-polymerase chain reaction (RT-PCR; Fig. 1D).

\section{Variable pattern defects in FoxH1 ${ }^{-/-}$embryos}

To characterize the embryonic lethality of the homozygous FoxH1 null allele, we analyzed between embryonic day 7.0 (E7.0) and E11.5 litters produced from heterozygote intercrosses. FoxH1 ${ }^{-/-}$embryos showed a variable phenotype that could be classified into three types. Embryos with the type I (least severe) phenotype show marked axial defects, lacking a definitive node and notochord. Embryos with the type II (intermediate) phenotype completely lack anterior structures but possess posterior structures with midline defects. Embryos with the type III (most severe) phenotype lack structures derived from the embryo proper as a result of A-P patterning defects; they thus resemble Cripto mutant embryos (Ding et al. 1998).

The three types of FoxH1-/- embryos were distinguishable morphologically at E8.5 (Fig. 2). Type I embryos (65/

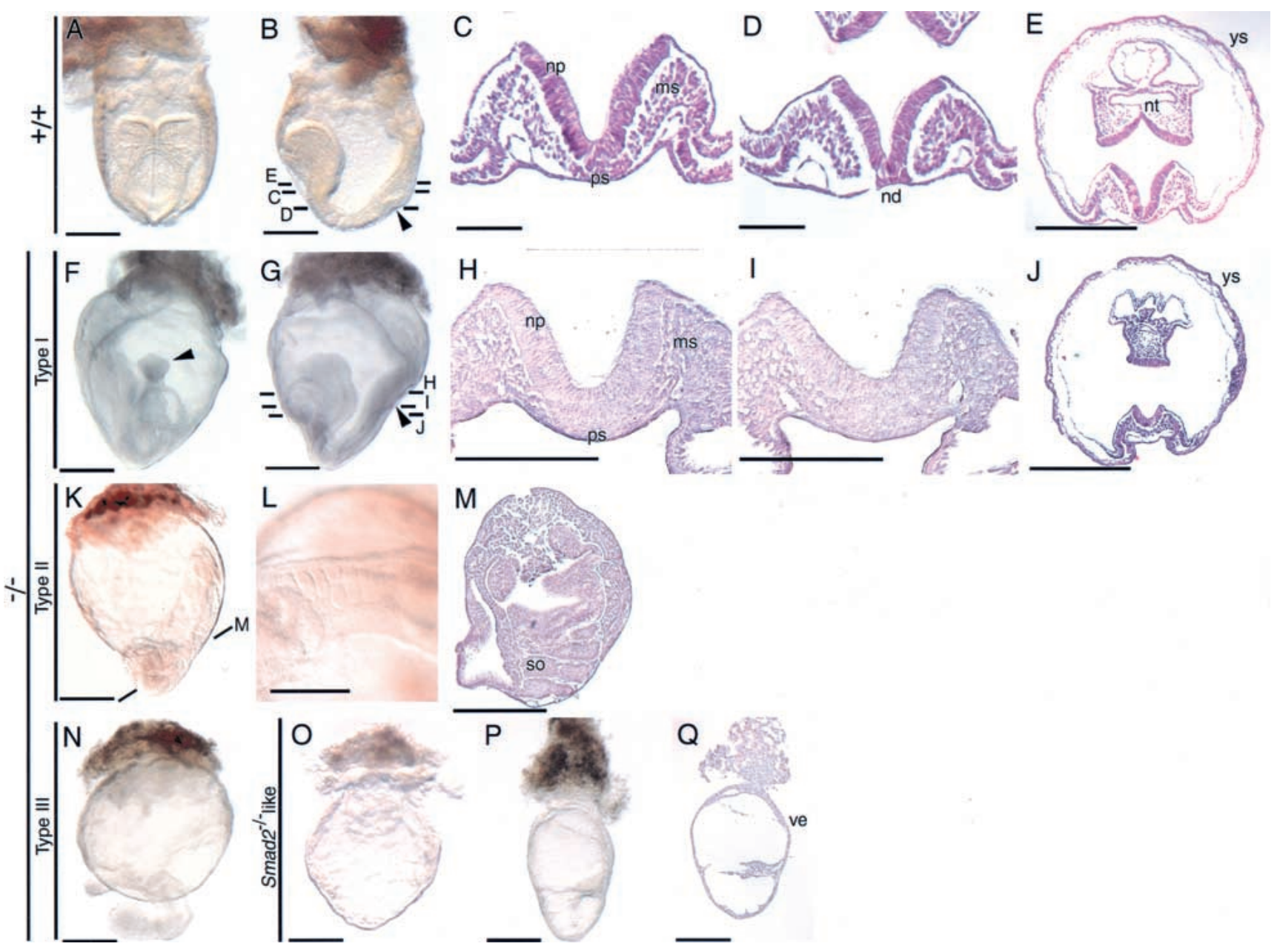

Figure 2. Three types of FoxH1-/- embryos at E8.5 $(A-N)$. Typical morphologies of wild-type $(+/+)$ embryos and of three types of FoxH1 $1^{-1-}$ mutant embryos at E8.5. Anterior views are shown in $A, F$, and $N$, whereas lateral views are shown in $B, G$, and $K$. The arrowhead in $B$ indicates the position of the node. Although the type I embryo lacks a node, the corresponding position is indicated by the arrowhead in $G$. The arrowhead in $F$ denotes a fused single head, which is characteristic of type I mutant embryos. A close-up ventral view of the type II embryo in $K$ is shown in $L$, highlighting the fused somites. Sections of the wild-type and type I embryos are shown in $C-E$ and $H-I$, respectively. The plane of each section is indicated in $B$ and $G$. Sections $C$ and $H$ are at the level of the primitive streak. Sections $D$ and $I$ are at the level of the node. Transverse sections were all oriented with anterior to the top. The type I embryo possesses a primitive streak $(H)$ but lacks a node $(I)$ and axial tissues $(J)$. Fused somites of a type II embryo are apparent in the section shown in $M$. The embryos shown in $O-Q$ are rare examples of the most severe (Smad2-/--like) phenotype observed for FoxH1 ${ }^{-/-}$ embryos at E8.5 $(O)$ or E7.5 $(P, Q)$. A frontal section of the embryo in $P$ is shown in $Q$. Endoderm cells are abnormal in that those in the embryonic region are columnar, an extraembryonic characteristic $(P, Q)$. The embryonic ectoderm is absent, but mesoderm-like cells are present inside (Q). ms indicates mesoderm; nd, node; np, neural plate; nt, notochord; ps, primitive streak; so, somites; and ys, yolk sac. Scale bars indicate $500 \mu \mathrm{m}$ with the exception of those in $C, D, H$, and $I$, which represent $100 \mu \mathrm{m}$. 
152 of $\mathrm{FoxH}^{-/-}$embryos, 43\%) showed a single, narrowfused head structure, or pinheadlike morphology (Fig. $2 F, G)$. In transverse sections, the primitive streak was detected (Fig. 2H), but midline structures such as the node, prechordal plate, notochordal plate-notochord, and floor plate were missing (Fig. 2I,J). The neural plate was unfolded and markedly thickened (Fig. 2I,J), and ectopic ingression of mesoderm was apparent between the neural plate and the primitive endoderm (Fig. 2J). Somites had formed, but they were fewer in number than in wild-type embryos and were fused in the midline. Type II mutant embryos (31/152 of FoxH1-/- embryos, $20 \%$ ) showed severe anterior truncation; they completely lacked anterior structures, such as the head fold, and instead manifested accumulation of cells in the distal region (occasionally, a beating heart-like structure was observed). Extraembryonic tissues such as the amnion and allantois, which were absent in type III embryos, were properly formed (Fig. 2K). The posterior portion of the embryo was formed but showed severe midline defects. Thus, several somites had formed, but they were fused across the midline (Fig. 2K-M). Type III embryos (52/152 of FoxH1 ${ }^{-/-}$embryos, 34\%) showed no signs of development of the embryo proper and showed a balloon-like morphology at this time (Fig. 2N). The embryo proper was recognized as a small cell mass located outside the yolk sac. In contrast, the yolk sac appeared relatively normal; it consisted of endodermal and mesothelial layers and contained blood islands. However, other components of extraembryonic tissue, such as the chorion, amnion, and allantois, were not observed, suggesting that the A-P axis was not properly formed. Rare embryos (4/152 of FoxH1-/- embryos, 3\%) showed relatively normal extraembryonic components with no embryo proper (Fig. 2O-Q) and therefore resembled Smad2 mutant embryos (Waldrip et al. 1998).

Three types of FoxH1 $1^{-/-}$embryos were also distinguishable on the basis of their morphology at E7.5. At this stage, mutant embryos showed various degrees of constriction at the extraembryonic-embryonic junction (Fig. 3). Mutant embryos that showed this constriction also manifested histological anomalies. In normal embryos, endoderm cells in the extraembryonic region are cuboidal and contain apical vacuoles, whereas those in the embryonic region are squamous. In the mutant embryos with the most marked constriction, however, endoderm cells in both the embryonic and extraembryonic regions are cuboidal and contain apical vacuoles (Fig.

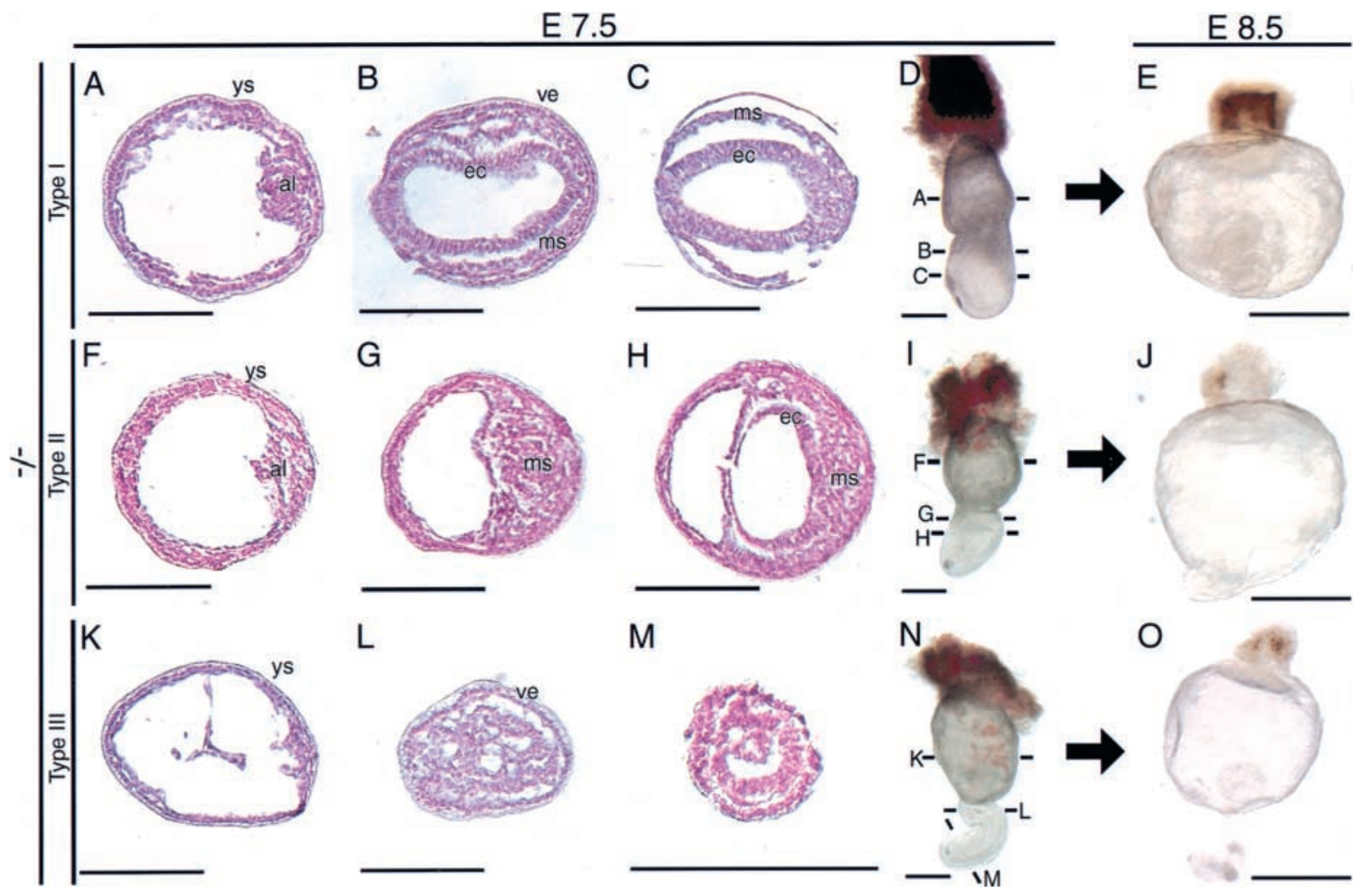

Figure 3. Morphology of FoxH1 $1^{-/-}$embryos at E7.5 and their subsequent development in vitro. Three types of $F O x H 1^{-/-}$embryos at E7.5 showed no $(D)$, mild $(I)$, or severe $(N)$ constriction at the extraembryonic-embryonic junction. Transverse sections at the level of the extraembryonic region $(A, F, K)$, the proximal embryonic region $(B, G, L)$, and the distal embryonic region $(C, H, M)$ are shown for each type of embryo. Embryos recovered at E7.5 were cultured in vitro for an additional $24 \mathrm{~h}(E, J, O)$. Embryos that showed no $(D)$, mild $(I)$, or severe $(N)$ constriction at E7.5 developed the type I $(E)$, type II $(J)$, and type III $(O)$ morphologies, respectively. Scale bars indicate 200 $\mu \mathrm{m}$ with the exception of those in $E, J$, and $O$, which represent $500 \mu \mathrm{m}$. al indicates allantois; ec, embryonic ectoderm; ms, mesoderm; ve, visceral endoderm; ys, yolk sac. 
$3 \mathrm{~K}-\mathrm{M})$. Furthermore, whereas the mesoderm layer was present in the extraembryonic region (Fig. 3K), the normal organized structure of the ectoderm and mesoderm was not apparent in the embryonic region (Fig. 3L,M). Such histological anomalies were not detected in FoxH1 ${ }^{-/}$embryos that did not show a constriction (Fig. 3A-C). Mutant embryos in which the constriction was apparent but not pronounced showed relatively organized structures, although mesodermal cells accumulated near the junction between the embryonic and extraembryonic regions (Fig. 3F-H).

To determine the relation between the defects apparent at E7.5 and those observed at E8.5, embryos were recovered at E7.5 and allowed to develop in vitro for an additional $24 \mathrm{~h}$. Embryos showing a severe constriction at E7.5 (Fig. 3N) developed the type III morphology after culture in vitro (Fig. 3O). Those showing a mild constriction (Fig. 3I) developed the type II morphology (Fig. 3J). Finally, FoxH1 ${ }^{-1-}$ embryos showing no constriction at E7.5 (Fig. 3D) developed the type I morphology (Fig. 3E). Therefore, embryos at stages earlier than E8.5 will hereafter also be referred to as type I, II, or III, accordingly.

\section{Impaired orientation of the A-P axis in type III}

$\mathrm{FoxH1}^{-/-}$embryos

The constriction at the extraembryonic-embryonic junction of type III (and, to a lesser extent, of type II) FOxH1 ${ }^{-/-}$ embryos at E7.5 is reminiscent of the constriction that is a characteristic feature of HNF3 $\beta / F O x A 2$ (Ang and Rossant 1994; Weinstein et al. 1994), Lim1 (Shawlot and Behringer 1995), Otx2 (Acampora et al. 1995; Matsuo et al. 1995; Ang et al. 1998), and nodal (Varlet et al. 1997) mutants, all of which show defects in anterior specification caused by impaired function of the anterior visceral endoderm (AVE). We therefore examined type III (and type II) embryos for several AVE markers.

In normal embryos at E5.5, two AVE marker genes, Hex and lefty1, are initially expressed in the visceral endoderm at the distal tip (Thomas and Beddington 1996; Thomas et al. 1998; data not shown). The visceral endoderm cells expressing these genes subsequently migrate anteriorly to form the AVE by E6.5. Thus, Hex, Cer-1, Hesx1, and lefty1 are all expressed in the AVE at E6.75 (Fig. 4A,D,H,N). In contrast, Brachyury is initially expressed in the proximal epiblast, but its expression domain subsequently moves to the posterior side at E6.5 and marks the primitive streak (Fig. 4A). These complementary cell movements establish A-P polarity.

In type III FoxH1 $1^{-/-}$embryos, however, Hex-expressing cells remained in the distal region at E6.75, with no evidence of movement toward the future anterior side (Fig. 4B). Cer-1 (Fig. 4E), Hesx1 (Fig. 4I), and Lim-1 (data not shown) were also expressed in the visceral endoderm at the distal tip at this time, indicating that the AVE is incorrectly formed in the distal region. Conversely, the Brachyury expression domain failed to move to the posterior side, remaining in the proximal epiblast (Fig. 4B,E,I,O). Otx2 expression in the visceral endoderm was maintained in the mutant embryo (Fig. 4L). The expres-

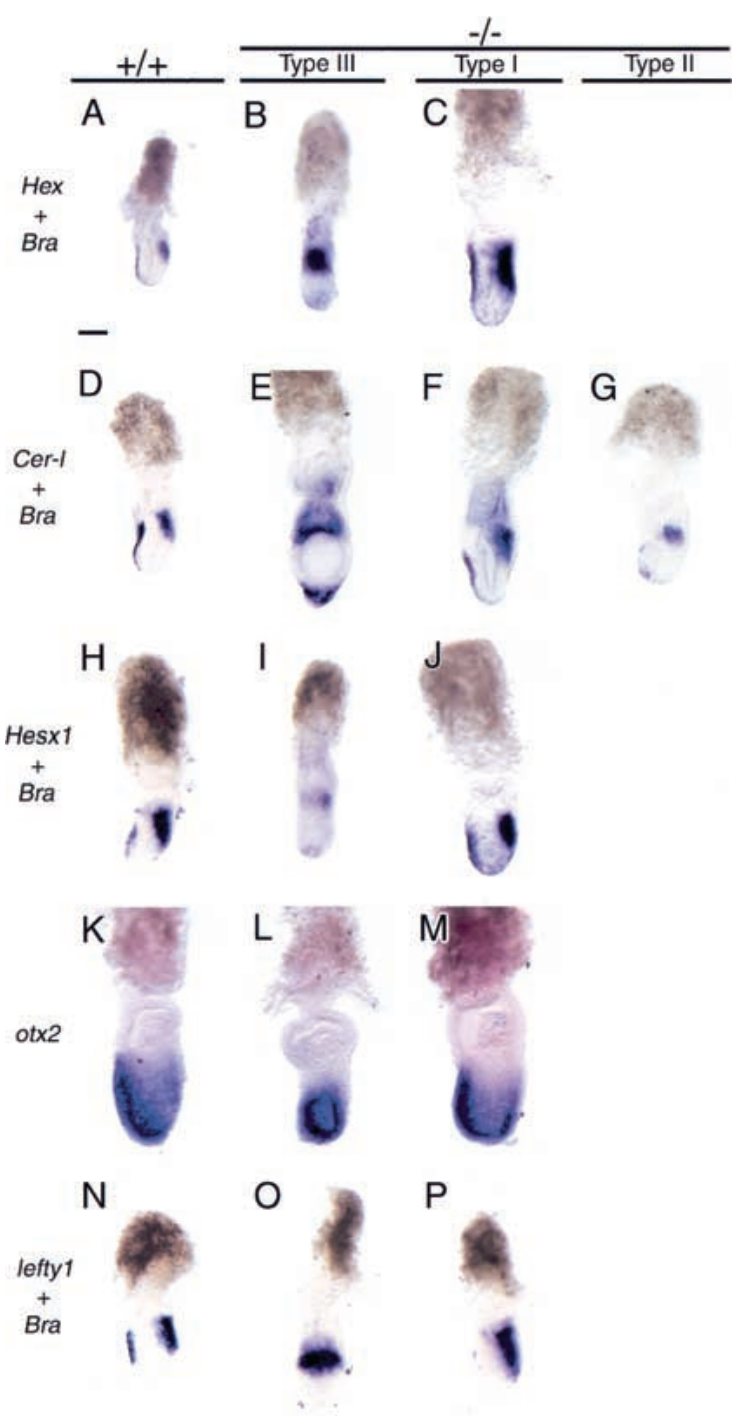

Figure 4. Misorientation of the A-P axis in type III FoxH1-/embryos. Expression of various AVE marker genes was examined by whole-mount in situ hybridization in wild-type $(+/+)$ embryos and in each of the three types of FoxH1 ${ }^{-1-}$ mutant embryos at E6.75. Probes were specific for Hex $(A-C)$, Cer-1 $(D-G)$, HesX-1 (H-J), Otx2 $(K-M)$ or lefty1 $(N-P)$ transcripts. The probe for Brachyury was also included in the indicated samples. Lateral views are shown for each embryo, with the anterior side on the left. Scale bars: $200 \mu \mathrm{m}$.

sion of lefty 1 in the visceral endoderm was abolished in the type III mutants (Fig. 4O), indicating that lefty1 expression in the endoderm may normally be induced by a Nodal-FoxH1 signaling pathway.

We also examined the type III embryo at E7.25. Whereas Brachyury expression remained in the proximal epiblast (Fig. 5C), expression of goosecoid, which marks the anterior primitive streak at this stage, was lost (Fig. 5I). FoxA2 expression, which also marks the anterior primitive streak, was absent, but its expression in the visceral endoderm was maintained (Fig. 5L,M). Furthermore, expression of lefty2, a marker for the nascent me- 


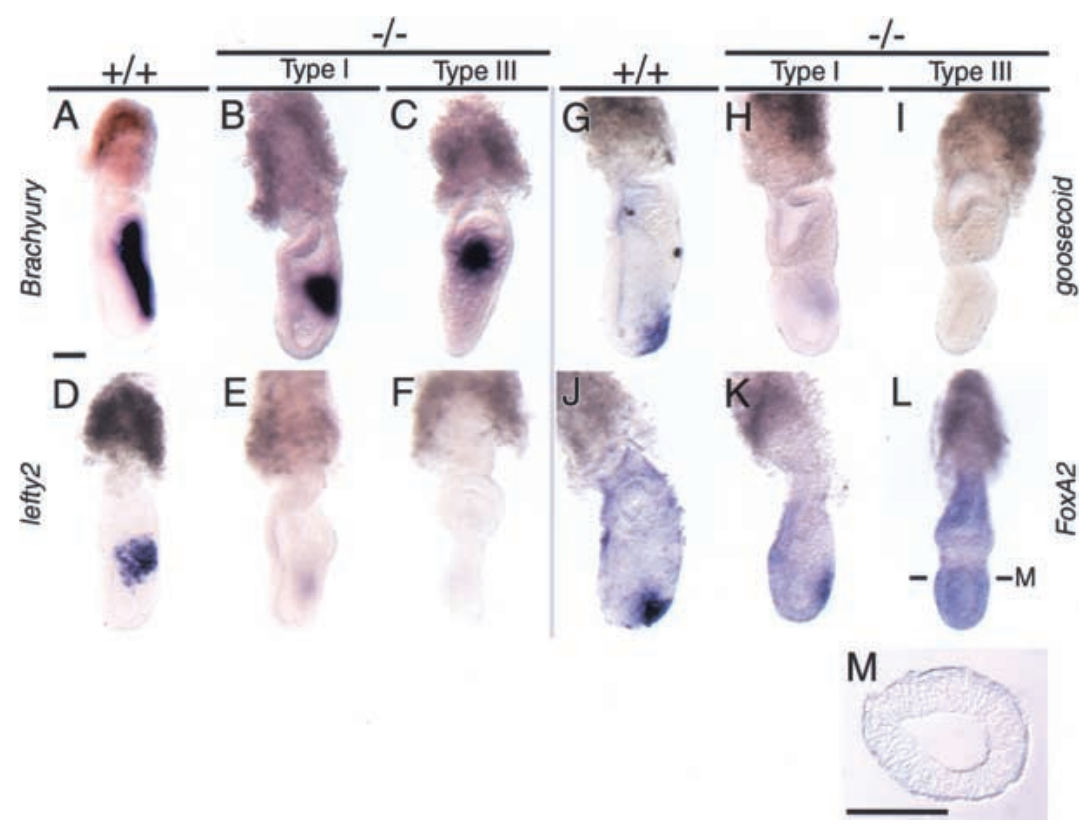

Figure 5. Defective morphogenesis of the primitive streak in FoxH1 $1^{-/-}$embryos. Expression of various marker genes for the primitive streak was examined by whole-mount in situ hybridization in wild-type $(+/+)$ embryos as well as in type III and type I FoxH1 $1^{-1-}$ embryos at E7.25. Probes were specific for Brachyury $(A-C)$, lefty2 $(D-F)$, goosecoid $(G-I)$, or FoxA2 $(J-L)$ transcripts. Lateral views are shown for each embryo, with the anterior side on the left. A transverse section at the plane indicated in $(L)$ are shown in $(M)$. FoxA2 expression was maintained in the endoderm of the type III mutant embryo. Scale bars: $200 \mu \mathrm{m}$. soderm, was also absent (Fig. 5F), indicating the lack of the primitive streak. Otx2, which is initially expressed before gastrulation throughout the epiblast and becomes restricted to the anterior third of the embryo by E7.5 (Ang et al. 1994), was widely expressed in the ectoderm layer of type III embryos at E7.5 (data not shown). These results indicate that proximal-distal (P-D) polarity is properly established in the type III mutants but that this polarity is not converted to the A-P axis. Incorrect orientation of the A-P axis is likely caused by impaired movement of the distal visceral endoderm. Consistent with this notion, sagittal sections of type III embryos (such as the one shown in Fig. 4E) revealed a marked accumulation of endoderm-like cells at the distal tip (data not shown). These defects are highly similar to those of Cripto mutants (Ding et al. 1998), but with one important difference: Anterior neural fates (Bf1- and En1-expressing cells) are induced in the distal region of Cripto mutants (Ding et al. 1998) but not in the corresponding region of type III FoxH1 ${ }^{-/}$embryos (data not shown). The visceral endoderm cells at the distal end are enlarged in Cripto mutants (Ding et al. 1998), whereas massive endoderm cells accumulate in type III $\mathrm{FoxH}^{-/-}$ mutants (Fig. 4E).

Type II embryos show similar but less severe phenotypes at this stage of development. The expression of Cer-1 in the visceral endoderm at E6.75 was down-regulated; the expression domain of this gene was apparent on the future anterior side but was located closer to the distal tip than in wild-type embryos (Fig. 4G). These results indicate that A-P patterning is partially impaired and that the AVE is not fully functional in type II embryos, which likely explains why they lack anterior structures. In contrast, type I embryos showed normal expression patterns for Brachyury (Fig. 4C,F,J,P), Hex (Fig. 4C), Cer-1 (Fig. 4F), Hesx1 (Fig. 4J), and Otx2 (Fig. $4 \mathrm{M}$ ) but had lost lefty1 expression in the AVE (Fig. 4P).
Failure of primitive streak elongation and lack of the node in type I FoxH1-/- mutants

Histological examination indicated that type I FoxH1-/embryos lack a definitive node, prechordal plate, and notochordal plate-notochord (Fig. 2H-J). To confirm these observations, we examined the expression at E8.5 of Shh, Brachyury, and HNF3 $\beta$ genes that are normally expressed in the node and its derivatives at this stage (Fig. $6 \mathrm{~A}, \mathrm{C}, \mathrm{E}, \mathrm{I})$. In type I mutants, the expression of Shh (Fig. 6B,D) and HNF3 $\beta$ (Fig. 6J) was completely lost, and only sparse expression of Brachyury was apparent at the anterior midline (Fig. 6F), indicating a deficiency of nodederived tissues. Type I embryos develop a single fusedhead structure (pinhead) and specifically lack the most rostral portion, the forebrain. Thus, Six3 expression, which is a marker for the forebrain (Fig. 6L), was abolished in type I mutants (Fig. 6M), whereas Otx2 expression, which marks the forebrain and midbrain (Fig. 6N), was detected in these mutants (Fig. 6O). In normal embryos, Fgf8 is expressed in the forebrain, midbrain-hindbrain junction, and posterior streak at this stage (Fig. 6C; Crossley and Martin 1995). In type I embryos, however, Fgf8 expression in the most anterior region was not apparent, although the other expression domains were preserved (Fig. 6H). The lack of the forebrain is likely because of the absence of the prechordal plate, insufficient function of the AVE, or both. Truncation of anterior structures is more severe in type II embryos. Thus, Otx2 expression (Fig. 6P) and HNF3 $\beta$ expression in the midline (Fig. 6K) are absent.

To investigate the mechanisms by which the absence of FoxH1 results in the failure of node formation, we examined the primitive streak of type I embryos at earlier stages. During normal gastrulation, Brachyury is expressed in the entire primitive streak, and its expression domain extends anteriorly with the extension of the 


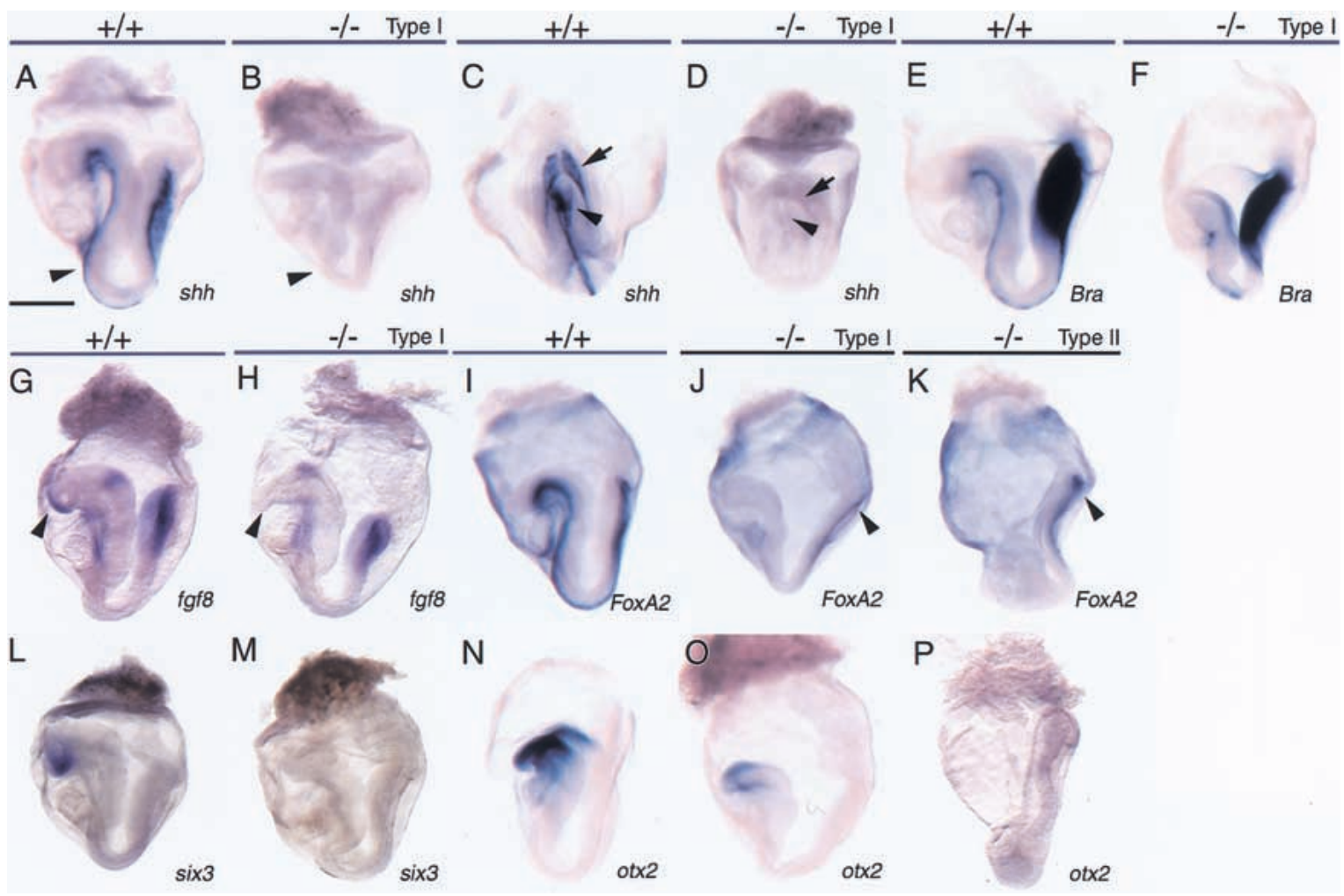

Figure 6. Midline defects in type I FoxH1 $1^{-/-}$embryos. Expression of various midline markers and forebrain markers in wild-type (+/+) and FoxH1 $1^{-1-}$ mutant embryos was examined at E8.5 by whole-mount in situ hybridization. Probes were specific for $S h h(A-D)$, Brachyury $(E, F)$, Fgf8 $(G, H)$, FoxA2 $(I-K)$, Six3 $(L, M)$, or Otx2 $(N-P)$ transcripts. All mutant embryos are type I with the exception of those shown in $K$ and $P$, which are type II. Lateral views are shown for each embryo with the anterior side on the left, except that posterior views are shown for $C$ and $D$. The arrowheads in $A$ and $B$ indicate the notochord. The arrowheads and arrows in $C$ and $D$ indicate the node and definitive endoderm, respectively. The arrowhead in $G$ indicates an $F g f 8$ expression domain in the forebrain, which was lost in $H$. In type II $(K)$ and type I $(J)$ mutant embryos, FoxA2 expression is absent in the definitive endoderm but is apparent in the yolk sac (arrowheads). Scale bars: $500 \mu \mathrm{m}$.

streak (Fig. 5A; Kispert and Herrmann 1994). In type I mutants, the Brachyury expression domain was localized to the posterior side, but it failed to elongate anteriorly or distally (Fig. 5B). The expression of goosecoid and FoxA2, which marks the anterior primitive streak at this stage in normal embryos (Fig. 5G,J; Sasaki and Hogan 1993; Ang and Rossant 1994; Weinstein et al. 1994), was also examined. In the mutant embryos, goosecoid expression was greatly reduced and observed in the posterior-proximal region of the embryo proper (Fig. 5H). The expression of FoxA2 was also down-regulated and detected in the proximal region of the embryo proper (Fig. 5K), indicating that the anterior primitive streak was not properly specified. The expression of lefty2, which marks nascent mesoderm generated from the primitive streak (Fig. 5D; Meno et al. 1997), was also markedly down-regulated in type I mutants (Fig. 5E). Given that lefty2 expression is induced by a NodalFoxH1 signaling pathway, at least in the lateral plate at the early somite stage (Saijoh et. al. 2000), this downregulation of lefty2 may result directly from the loss of Nodal signaling. Together, these results indicate that formation of the primitive streak is initiated in type I embryos, but the anterior portion of the streak is not properly specified, resulting in node agenesis.

\section{Genetic interaction between FoxH1 and nodal}

The expression of nodal is induced by a Nodal-FoxH1 signaling pathway, at least in certain aspects of development, such as left-sided expression of this gene in the lateral plate at the early somite stage (Osada et al. 2000; Saijoh et al. 2000). Expression of nodal in the posteriordistal ectoderm during gastrulation also may be regulated by a FoxH1-dependent enhancer (Norris and Robertson 1999). We therefore examined nodal expression in FoxH1 $1^{-/}$embryos with the use of in situ hybridization. In wild-type embryos, this gene is initially expressed throughout the epiblast and in the underlying primitive endoderm at E5.5, but its expression in the epiblast becomes progressively restricted to the posterior region (Fig. 7A; Varlet et al. 1997). The expression of nodal disappears from the AVE at E6.75, but it is maintained in the lateral and posterior portions of visceral endoderm. It disappears from the posterior ectoderm and the visceral endoderm at E7.25 and then begins in the prospective 


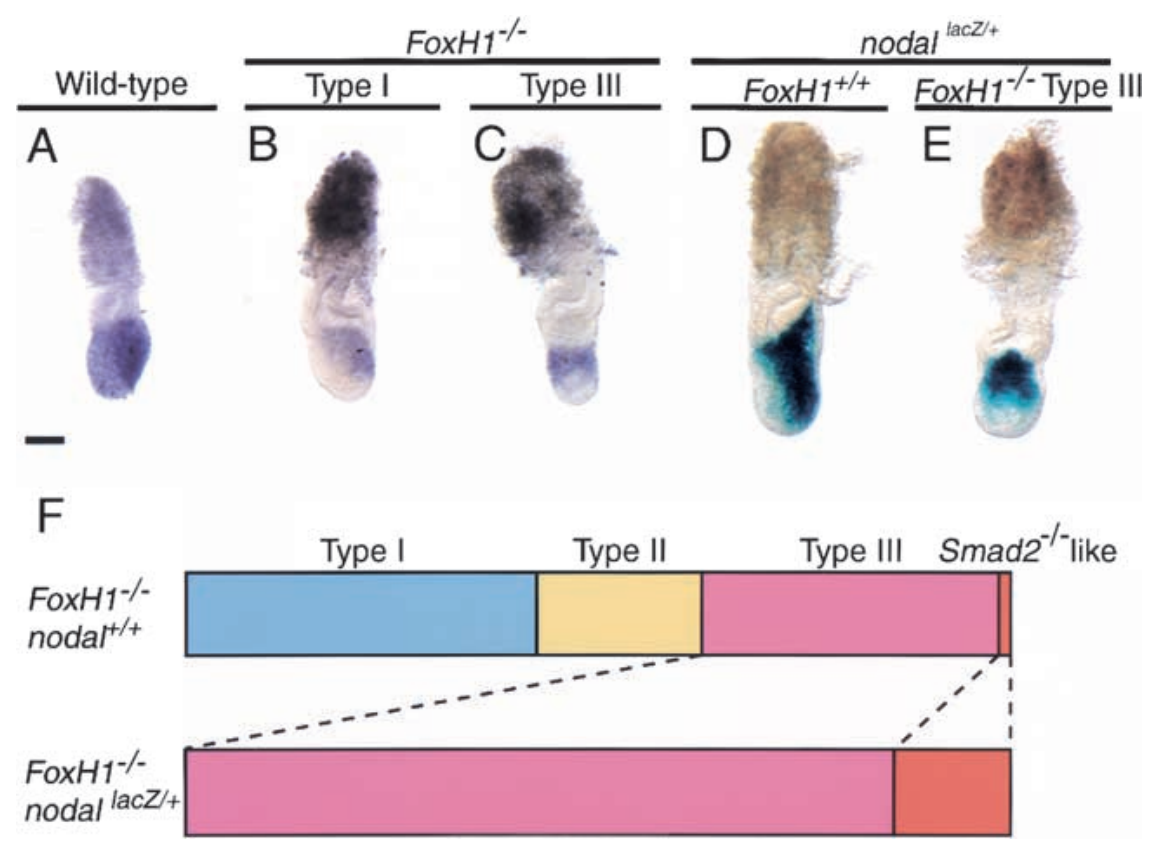

Figure 7. Genetic interaction between FoxH1 and nodal. The expression of nodal in wild-type $(A)$, type I FoxH1-/- $(B)$, and type III FoxH1-l- $(C)$ embryos was examined at E7.0 by whole-mount in situ hybridization. In type I embryos, nodal expression was greatly reduced and was confined to the proximal posterior ectoderm $(B)$. In type III embryos $(C)$, nodal expression was down-regulated and remained at the rim of the proximal epiblast. The frequencies of type III, type II, type I, and Smad2-/--like phenotypes were also determined for FoxH1 ${ }^{-/-}$, nodal $^{+/+}$(upper bar) and FoxH1 ${ }^{-/-}$,nodal lacZ/+ (lower bar) embryos at E8.5 $(F)$. The total numbers of the embryos examined are 152 and 28 for FoxH1 $1^{-/-}$, nodal $^{+/+}$and FoxH1 $1^{-/-}$, nodal ${ }^{\text {lac }} /+$, respectively. Most FoxH1 $1^{-/-}$,nodal ${ }^{\mathrm{lac} /+}$ embryos showed the type III morphology. Staining for $\beta$-galactosidase activity is also shown for FoxH1 $1^{+/+}$,nodal lacZ/+ $(D)$ and FoxH1 $1^{-/-}$, nodal lacZ/+ $(E)$ embryos at E7.0. Scale bars: $200 \mu \mathrm{m}$. node. In type III FoxH1-/- embryos, however, nodal expression was down-regulated and remained at the rim of the proximal epiblast at E7.0, without being shifted to the posterior side (Fig. 7C). In type I embryos, the abundance of nodal mRNA was also reduced (Fig. 7B); the nodal expression site was shifted to the posterior side but was localized more proximally than in wild-type embryos. The down-regulation of nodal apparent in FoxH1-l- embryos indicates that the expression of this gene in the epiblast is maintained by a positive autoregulatory loop that includes FoxH1.

We also examined the potential genetic interaction between FoxH1 and nodal by crossing FoxH1 and nodal lacZ mutants (Collignon et al. 1996). Double heterozygotes $\left(\right.$ FoxH $^{+/-}$, nodal $\left.^{\text {lac } /+}\right)$ appeared normal and were crossed with FoxH1 ${ }^{+/-}$mice. The phenotype of the resulting FoxH1 $1^{-/-}$, nodal lacZ/+ embryos was more severe than that of $\mathrm{FoxH1}^{-/-}$, nodal ${ }^{+/+}$embryos (Fig. 7F). Thus, most $(24 / 28,86 \%)$ of the FoxH1 $1^{-/}$, nodal ${ }^{\text {lacZ/+ }}$ embryos examined at E8.5 were type III, manifesting A-P patterning defects; the remaining embryos $(4 / 28,14 \%)$ resembled Smad2 mutant embryos, similar to the FoxH1-/- mutant shown in Figure 2O. As expected, nodal expression, which was monitored on the basis of the activity of the nodal ${ }^{\text {lacz }}$ allele, remained in the proximal epiblast at E7.0 in all FoxH1 ${ }^{-/-}$, nodal ${ }^{\text {lacZ/+ }}$ embryos examined (Fig. $7 \mathrm{E})$.

Rescue of A-P patterning defects, but not midline defects, in FoxH1 ${ }^{-/-}$embryos by expression of FoxH1 in extraembryonic tissues

We next examined whether FoxH1 is required in the epiblast lineage or in the extraembryonic lineage, including the primitive endoderm. The FoxH1 gene was specifi- cally deleted from the epiblast with the use of the Fox $H 1^{\text {flox }}$ allele and transgenic mice that express Cre in the epiblast and its derivatives but not in the primitive endoderm. The Cre-expressing transgenic mice harbor lefty2-Cre, a fusion construct comprising the Cre gene linked to the $5.5-\mathrm{kb}$ upstream region of lefty2 (Fig. 8A). When linked to the lacZ gene, the $5.5-\mathrm{kb}$ upstream region of lefty2 confers expression in the nascent mesoderm at E6.5 to E7.0 and in the left lateral plate mesoderm at E8.25 (Saijoh et al. 1999). However, one lefty2Cre transgenic line (21B) showed epiblast-specific expression of Cre between E5.5 and E8.0 (lefty2 is not expressed in the epiblast). Thus, crossing of line $21 \mathrm{~B}$ animals with mice that harbor a Cre-sensitive lacZ reporter gene (Sakai and Miyazaki 1997) yielded embryos harboring both lefty2-Cre and the lacZ reporter gene that showed $\beta$-galactosidase activity throughout the epiblast lineage but not in the extraembryonic tissues, including the primitive endoderm, both at E6.5 (Fig. 8B,C) and E7.0 (Fig. 8D,E).

We crossed $\mathrm{FoxH}^{+/-}$animals harboring the Cre transgene with FoxH1 ${ }^{\text {flox/flox }}$ mice and genotyped the resulting embryos by PCR analysis of yolk sac DNA. FoxH $1^{\text {flox/- }}$, lefty2-Cre embryos were first examined at E8.5; most $(15 / 16,94 \%)$ of these embryos showed the type I phenotype, having a single fused head (Fig. 8J-L).

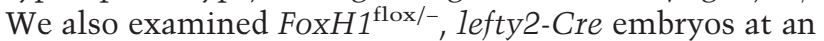
earlier stage (E7.0). Again, they (3/3) showed the type I morphology, showing normal Cer-1 expression in the AVE region (Fig. $8 \mathrm{~N}$ ); type III embryos showing Cer-1 expression at the distal end (such as the one shown in Fig. 4B) were not detected $(0 / 3)$ at this stage. Unexpectedly, lefty 1 expression in the visceral endoderm was lost in the epiblast-specific FoxH1 mutant embryos (Fig. 8P), indicating that the expression of lefty 1 is induced by 

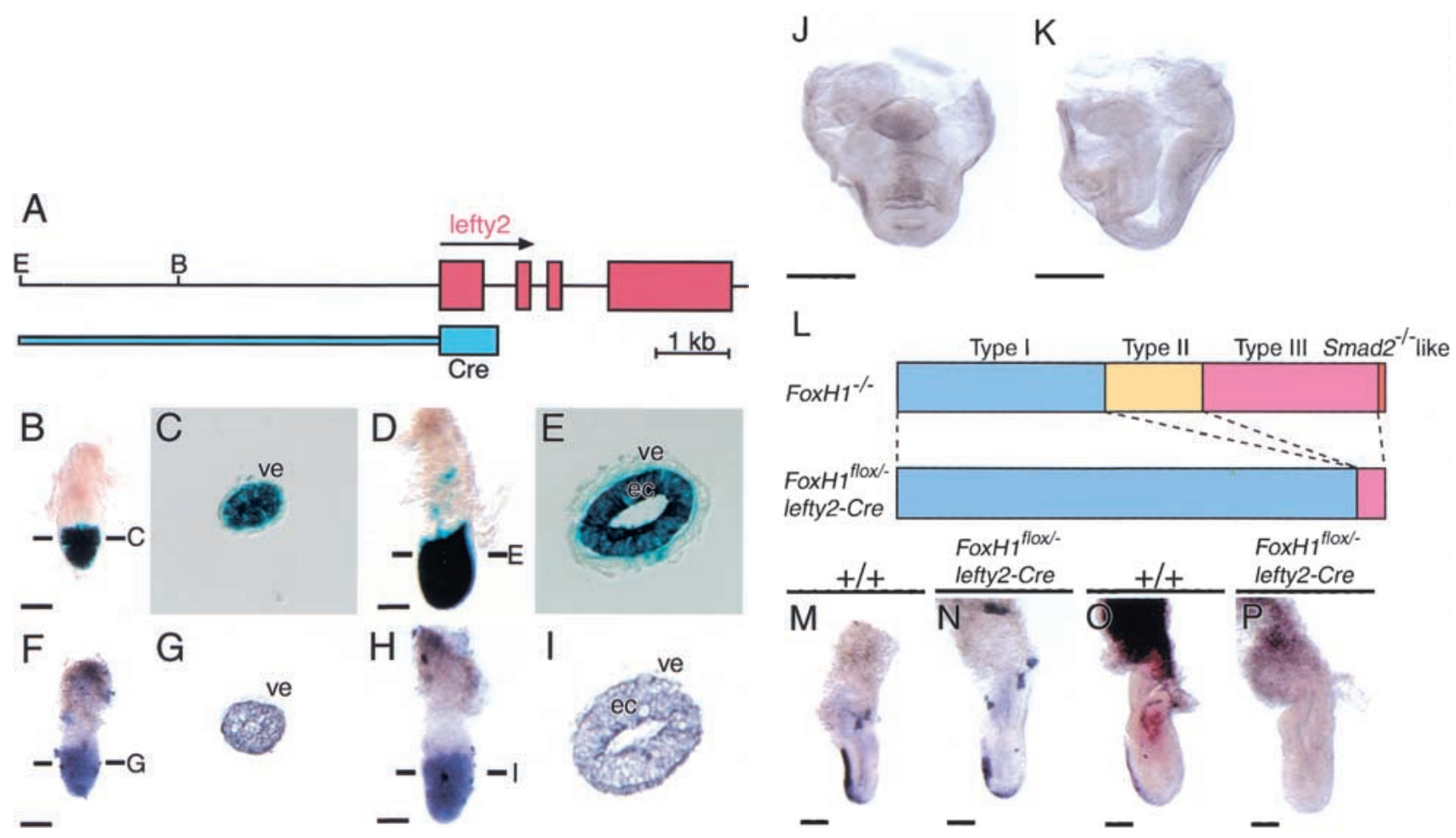

Figure 8. Midline defects but normal A-P patterning in embryos with epiblast-specific deletion of FoxH1. The structure of the lefty2-Cre transgene, in which Cre is linked to the $5.5-\mathrm{kb}$ upstream region of mouse lefty2, is shown in A. Mice harboring this transgene (line 21B) were crossed with mice that express lac $Z$ in response to Cre. Embryos containing both lefty2-Cre and the lacZ reporter gene were stained for $\beta$-galactosidase activity at E6.5 $(B, C)$ and $\mathrm{E} 7.0(D, E)$; transverse sections at the planes indicated in $B$ and $D$ are shown in $C$ and $E$, respectively. At both stages, staining is apparent in the epiblast but is absent from the remaining portions of the embryo. Expression of FoxH1 was examined in the wild-type embryos by whole-mount in situ hybridization at E6.5 (F,G) and E7.0 $(H, I)$; transverse sections at the planes indicated in $F$ and $H$ are shown in $G$ and $I$, respectively. A Fox $H 1^{\text {flox/- }}$ embryo harboring the lefty2-Cre transgene examined at E8.5 showed typical type I morphology, with a fused head $(J, K)$. Anterior and lateral views of the same embryo are shown in $J$ and $K$, respectively. The frequencies of type III, type II, type I, and Smad2 ${ }^{-/-}$-like phenotypes are shown for FoxH1 $1^{-1-}$ (upper bar) and FoxH1 $1^{\text {flox/- }}$ lefty2-Cre (lower bar) embryos at E8.5 (L); most of the FoxH1 $1^{\text {flox/- }}$, lefty2-Cre embryos showed the type I morphology. The total number of the FoxH1 flox/-, lefty2-Cre embryos examined is 16. Expression of Cer-1 (M,N) and lefty1 $(O, P)$ was examined in wild-type $(+/+)$ and FoxH1 $1^{\text {flox } /-}$, lefty2-Cre embryos at E7.0; the FoxH $1^{\text {flox } /-}$, lefty2-Cre embryos showed a normal Cer-1 expression pattern $(N)$ but had lost lefty1 expression in the AVE $(P)$. Scale bars indicate $200 \mu \mathrm{m}$ with the exception of those in $J$ and $K$, which represent $500 \mu \mathrm{m}$. ec indicates embryonic ectoderm; ve, visceral endoderm.

FoxH1-dependent signals (most likely, Nodal signals) derived from the epiblast. Type II embryos were not detected at either E8.5 or E7.0. These results indicate that the presence of FoxH1 in the primitive endoderm is able to rescue the A-P patterning defects of type III and type II embryos but not the anterior primitive streak defects of type I embryos.

We also examined chimeric embryos that were generated by injecting $\mathrm{FoxH}^{+/+}$ES (ROSA26) cells into FoxH $1^{-/-}$blastocysts (Fig. 9). Among such chimeras with extensive colonization of ROSA26 ES cells, about a half of them (5/9) showed severe A-P patterning defects characteristic of type III phenotype (Fig. 9J,K). In the remaining chimeric embryos, midline defects were mostly rescued (Fig. 9E-I). Thus, the node and notochordal plate were formed (data not shown), and somites on the both sides were separated by the midline (Fig. 9I). The neural plate was folded along the A-P axis (Fig. 9G), but the most rostral part of the neural plate remained unfolded
(Fig. 9H). Therefore, injection of wild-type ES cells could rescue midline defects but failed to rescue A-P patterning defects.

These results indicate that FoxH1 in the primitive endoderm is required for A-P patterning, whereas FoxH1 in the epiblast is essential for primitive streak formation. The expression pattern of FoxH1 is consistent with this notion; FoxH1 was expressed both in the visceral endoderm and in the epiblast, but not in the extraembryonic region, of wild-type embryos at E6.5 to E7.0 (Figs. 8F-I). Therefore, among the nonepiblast tissues, it is most likely in the visceral endoderm that FoxH1 plays a role in A-P patterning.

\section{Discussion}

Our analysis of FoxH1 mutant mice indicates that FoxH1 is the major transcriptional transducer of Nodal signaling in early development. This transcription factor 


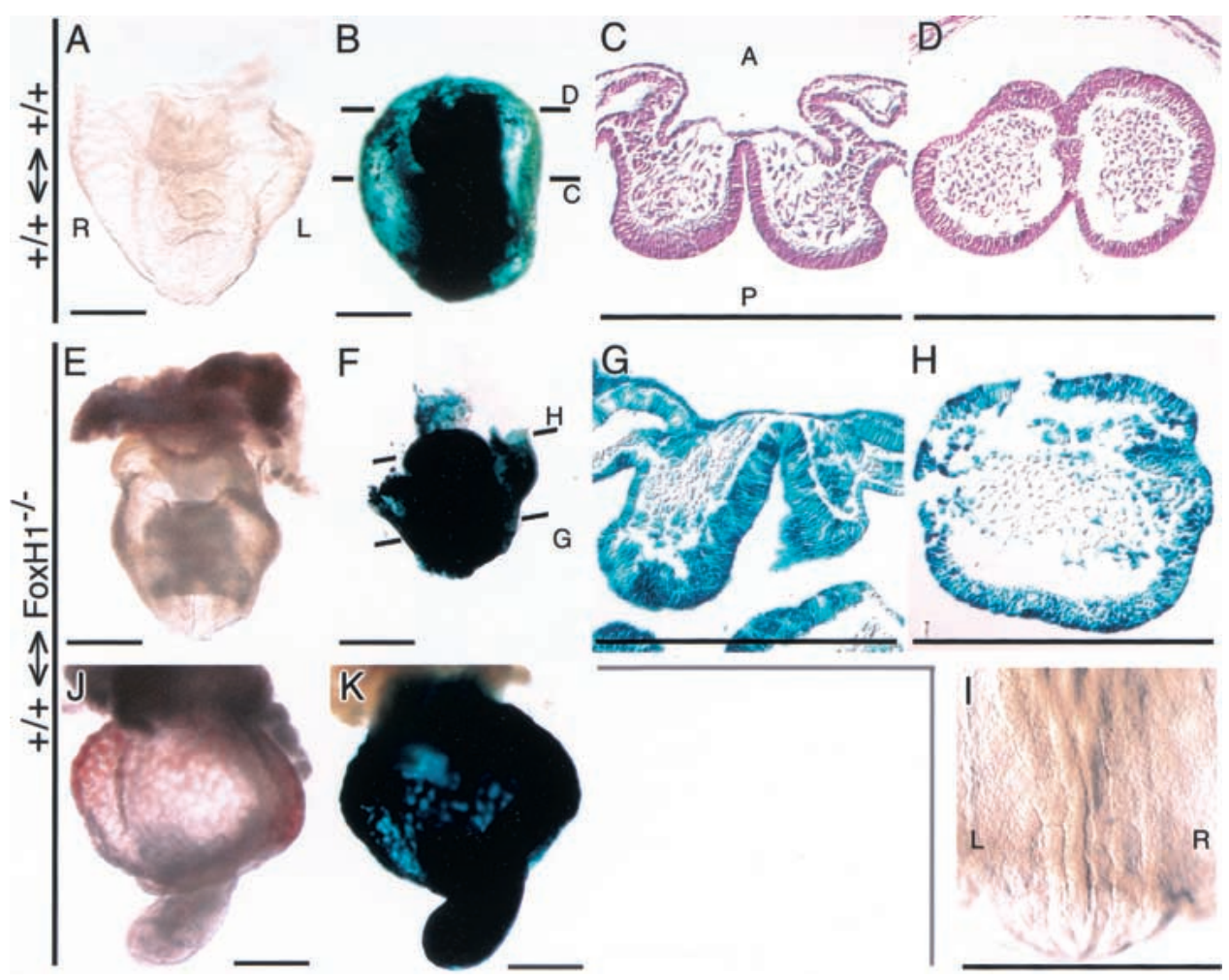

Figure 9. Rescue of midline defects but not A-P patterning defects by wild-type embryonic cells in wild-type $(\leftrightarrow)$ FoxH1 $1^{-/-}$chimeric embryos. Wild-type $(\leftrightarrow)$ FoxH1 $1^{+/}(A-D)$ and wild-type $(\leftrightarrow)$ FoxH1 $1^{-/-}(E-K)$ chimeric embryos were recovered at E8.5. Whole-mount views of the chimeric embryos with extensive colonization of wild-type ES cells are shown before $(A, E, I, J)$ or after $(B, F, K) \mathrm{X}$-Gal staining. They are all anterior views except that a ventral view is shown in $I$. Hematoxylin and eosine-stained sections of a wild-type $(\leftrightarrow)$ Fox $H 1^{+/+}$chimeric embryo at the levels of the trunk and forebrain are shown in $C$ and $D$, respectively. Corresponding sections of X-Gal stained wild-type $(\leftrightarrow)$ FoxH1 $1^{-/-}$chimeric embryo are shown in $G$ and $H$. About a half of the wild-type $(\leftrightarrow)$ FoxH1 $1^{-/-}$embryo shown in $E-I$, midline defects were rescued $(E, G, I)$ whereas the forebrain remained abnormal $(H)$. The neural plate, except for the most rostral part $(H)$, was folded normally $(G)$. The remaining wild-type $(\leftrightarrow) F O x H 1^{-1-}$ embryos $(J, K)$ showed severe A-P patterning defects characteristic of type III phenotype. Scale bar indicates $500 \mu \mathrm{m}$.

appears to play multiple roles: Its activity in the primitive endoderm and in the epiblast is essential for A-P patterning and for node formation, respectively. A zebrafish mutant (schumalspur, or sur) that is deficient in FoxH1 has been described recently (Pogoda et al. 2000; Sirotkin et al. 2000). This mutant lacks an organizer and shows defects in dorsal axial structures that are equivalent to the defects observed in type I FoxH1-/- mice.

\section{FoxH1-dependent signals in visceral endoderm are required for orienting the A-P axis}

The A-P axis is established by three sequential steps: (1) graded expression of several genes along the P-D axis of the embryo, (2) movement of the distal visceral endoderm toward the anterior end of the embryo, and (3) specification of the underlying epiblast to an anterior identity by AVE-derived signals (Beddington and Robertson 1998, 1999; Kimura et al. 2000).

In type III FoxH1 ${ }^{-/-}$embryos, the P-D axis is established properly in the egg cylinder structure, but the distal visceral endoderm fails to migrate anteriorly. Epiblast-specific deletion of FoxH1 indicated that FoxH1 in the visceral endoderm is essential for cell movement. The FoxH1-dependent signals may be provided by Nodal, a notion that is supported by previous observations by other researchers. Thus, type III FoxH1 mutants show A-P patterning defects similar to those of mouse mutants that lack Cripto (Ding et al. 1998), which functions as a coreceptor for Nodal (Sakuma et al., in prep.). Furthermore, chimeric embryos consisting of wild-type epiblast and $A L K 4^{-/-}$endoderm (Gu et al. 1998) fail to undergo gastrulation and show defects similar to those of type III FoxH1 mutants (ALK4 is most likely a type I receptor for Nodal; R. Sakuma, Y. Ohnishi, C. Meno, and H. Hamada, in prep.). The mechanism by which FoxH1dependent signals (Nodal signals) promote the anteriordirected migration of the distal visceral endoderm remains unknown. One possibility is that Nodal activity may be distributed unevenly along the future A-P axis in the region of the distal visceral endoderm, and this differential Nodal activity may generate differences in cell proliferation or in the orientation of cell division.

Formation of the anterior neural structures requires one additional step: stabilization of anterior identity by signals derived from the prechordal plate (Rhinn et al. 
1998; Shawlot et al. 1999; Tam and Steiner 1999). The absence of the forebrain in type I FoxH1-/- embryos may result from the lack of the prechordal plate, which comprises axial mesoderm cell populations derived from the anterior streak. Consistent with this conclusion, epiblast-specific deletion of FoxH1 was sufficient to give rise to type I embryos lacking the forebrain. However, impairment of AVE function can also induce a similar phenotype, as evident in chimeric nodal ${ }^{-/-}$embryos that show extensive colonization of wild-type ES cells (Varlet et al. 1997). Therefore, the absence of the forebrain in type I embryos might also result from dysfunction of the AVE. Indeed, the forebrain was also impaired in chimeric embryos in which the visceral endoderm is composed of FoxH1 $1^{-/-}$cells.

In an accompanying paper, Hoodless et al. (2001) also report FoxH1 mutant mice, which showed similar phenotype. However, there was a difference in the degrees of the A-P patterning defects. In their mutant mice, the prospective AVE cells can move anteriorly, although this migration may be delayed. Thus, their mutants do not show A-P patterning defects at later stages. The most likely reason for this difference is genetic background. Our mutant mice have a 129/B6 mixed background, whereas their mutant mice have a $129 / \mathrm{CD}-1$ mixed background. Genetic interaction between FoxH1 and nodal indicates that both FoxH1-dependent and independent pathways mediate Nodal signaling during A-P patterning. Perhaps, FAST-independent pathway can complement the absence of FoxH1 in some genetic backgrounds but not in others.

\section{Role of FoxH1 in formation and patterning of the primitive streak}

Despite extensive studies in various vertebrates, little is known of the mechanism by which formation of the primitive streak is initiated in the mouse. The observations that mice with mutations in nodal or in genes for its putative receptors, such as ActRIB (ALK4), ActRIIA, and $A c t R I I B$, fail to gastrulate (Conlon 1994; Gu et al. 1998; Song et al. 1999/ indicate that Nodal signals are essential for this process. However, type I and type II FoxH1-/- embryos formed the primitive streak, and posterior development was relatively normal in these animals, suggesting that FoxH1 is dispensable for streak formation. Nodal signaling is thus likely mediated by transcription factors other than FoxH1 during streak formation.

The primitive streak is initially formed in a small region near the extraembryonic-embryonic junction and elongates distally during gastrulation. The streak is patterned along the A-P axis, and cells derived from this structure are allocated to various mesodermal lineages, depending on their stage and position. For instance, cells derived from the early streak contribute predominantly to extraembryonic mesoderm, and the anterior portion of the mid-to-late primitive streak contributes to the node (Lawson et al. 1986; Tam and Beddington 1987). How- ever, little is known of the mechanisms that underlie these patterning events.

The primitive streak of type I Fox $\mathrm{H1}^{-/-}$mutants failed to elongate and lacked the anterior portion. The truncation of the streak in these embryos is likely because of down-regulation of nodal expression in the posterior ectoderm. Thus, nodal expression was abolished in the distal-posterior epiblast and was markedly reduced in the proximal-posterior region of the epiblast in the type I mutants. These observations on nodal expression are consistent with the results of recent studies on the transcriptional regulation of nodal (Adachi et al. 1999; Norris and Robertson 1999). The expression of nodal in the ectoderm is controlled by at least two enhancers: The FoxH1-dependent enhancer (referred to as ASE) induces expression in the posterior ectoderm, whereas the other enhancer induces expression in the proximal epiblast. The lack of FoxH1 would therefore be expected to reduce nodal expression in the posterior ectoderm. In conclusion, FoxH1 is not essential for the initiation of primitive streak formation. However, it plays an important role in elongation and patterning of the streak; specifically, it maintains nodal expression in the anterior portion of the streak by acting as a component of a Nodal autoregulatory loop.

In other vertebrates, such as Xenopus and the chicken, the organizer is induced by synergistic stimulation by Wnt and Nodal-Activin-like signals (Harland and Gerhart 1997). In frog and zebrafish, a Nodal-FoxH1 pathway induces the expression of organizer-associated genes (Toyama et al. 1995; Watanabe and Whitman 1999). Furthermore, FoxH1 mutants (sur) in zebrafish fail to form a gastrula organizer (Pogoda et al. 2000; Sirotkin et al. 2000). These observations and our analysis of FoxH1 mutant mice indicate that a Nodal-FoxH1 signaling pathway plays a conserved role in organizer formation in vertebrates.

\section{FoxH1 mediates Nodal signaling during early mouse development}

FoxH1 was initially identified as a mediator of Activin signaling (Chen et al. 1996). In cultured cells or frog animal caps, FoxH1 interacts with Smad2 (or Smad3) and Smad 4 and mediates signaling by TGF- $\beta$ and TGF- $\beta$-related factors such as Activin and Nodal (Chen et al. 1996; Labbe et al. 1998; Weisberg et al. 1998; Zhou et al. 1998; Liu et al. 1999; Saijoh et al. 2000). However, our data now indicate that FoxH1 plays the major role in mediating Nodal signaling during early development of the mouse. FoxH1-/- mice showed various patterning defects that have been observed previously in mutant mice lacking other components of the Nodal signaling pathway. Thus, type III FoxH1-l- embryos manifested A-P patterning defects similar to those apparent in Cripto mutants (Ding et al. 1998). In addition, similar to Cripto $^{-/-}$mice (Ding et al. 1998) and Smad2-/- chimeric mice (Tremblay et al. 2000), type II and type I FoxH1-/embryos lacked definitive endoderm. Furthermore, the phenotype of type I FoxH1 $1^{-/-}$embryos was similar to 
that of chimeric nodal ${ }^{-1-}$ embryos with a small contribution of wild-type ES cells (Varlet et al. 1997). Genetic interaction was apparent between FoxH1 and nodal. Consistent with the suggestion that expression of nodal and lefty2 is induced by a Nodal-FoxH1 pathway (Saijoh et al. 2000), we showed that nodal expression was initiated but down-regulated in type III and type I FoxH1 ${ }^{-/-}$ embryos and that lefty2 expression was markedly reduced in the type III and type I mutants. The expression of lefty 1 in the visceral endoderm also may be induced by a Nodal-FoxH1 pathway, given that expression of this gene in this region was lost in type III FoxH1 $1^{--}$embryos and epiblast-specific FoxH1 mutant embryos.

Despite the similarities between the phenotypes of FoxH1 $1^{-/-}$mutants and mutants lacking other components of the Nodal signaling pathway, substantial differences are also apparent. In Cripto mutants, the AVE incorrectly formed at the distal tip acts on the underlying epiblast and induces anterior neural fates in the absence of streak-derived tissues (Ding et al. 1998). In contrast, such anterior neural identity was not induced in type III FoxH1 $1^{-/-}$mutants. This apparent discrepancy is not easily reconciled, but one possible explanation is that Cripto plays a role in addition to functioning as a coreceptor for Nodal signaling. For instance, Cripto may confer lability on signals from the visceral endoderm, as suggested previously by others (Shawlot et al. 1999). The patterning defects of $\mathrm{FOXH1}^{-1-}$ mutants are less severe than are those of embryos completely lacking Nodal. Thus, the extraembryonic tissues are highly abnormal in nodal ${ }^{-1}$ mutants, whereas those tissues are relatively normal in type III FoxH1 mutants. The phenotype of FoxH1 $1^{-1-}$ mutants is also less severe than that of Smad2 mutants. In the absence of Smad2, embryos fail to establish P-D polarity properly, and the entire epiblast adopts an extraembryonic mesodermal fate (Waldrip et al. 1998; Heyer et al. 1999). In contrast, FoxH1 ${ }^{-/-}$embryos establish P-D polarity. These phenotypic differences among nodal, Smad2, and FoxH1 mutants suggest that Nodal signals act through both FoxH1-dependent and FoxH1independent pathways. The actions of Nodal in A-P patterning and in patterning and elongation of the primitive streak are FoxH1 dependent, whereas those in mesoderm induction are FoxH1 independent.

Nodal signaling is also implicated in left-right patterning at a later stage of development (Schier and Shen 2000), and FoxH1 may play a role in mediating Nodal signals during this process. However, the early death of FoxH1 mutant mice prevented us from studying the role of this transcription factor in left-right asymmetry. FoxH1 also may mediate signaling by TGF- $\beta$ and related factors at later stages of development. Clarification of the roles of FoxH1 at these later stages will require conditional deletion of the gene.

\section{Materials and methods}

\section{Generation of FoxH1-deficient mice}

Genomic FoxH1 clones were isolated from a genomic DNA library constructed from E14 ES cells. A targeting vector was constructed by subcloning the $5^{\prime}$ flanking region (the 3-kb SalISacII fragment), the exon 1 -intron 1 region (the $2.3-\mathrm{kb}$ SacIISmaI fragment), and the region containing the other exons and intron as well as the $3^{\prime}$ flanking region (the $7.8-\mathrm{kb} S \mathrm{maI}-\mathrm{XbaI}$ fragment) of FoxH1 into a modified pMC1-DTpA vector (Taniguchi et al. 1997). A loxP fragment containing a BamHI site was inserted between exon 1 and exon 2, and a loxP-Frtneo-Frt cassette (Meyers et al. 1998) was inserted into the SacII site in the $5^{\prime}$ flanking region. Gene targeting was performed as described (Sawai et al. 1991). The targeting vector was linearized with NotI before introduction into R1 ES cells by electroporation.

Of 140 G418-resistant ES clones, two clones (F94, F128) were shown to have undergone homologous recombination, as confirmed by Southern blot analysis with various probes, including a $5^{\prime}$ probe, a $3^{\prime}$ probe, and a neo probe. To generate a flox allele, we subjected F128 cells to electroporation with a Flp expression vector (pCAGGS-Flpe-IRES-puro; kindly provided by F. Stewart and S. Dymecki) followed by selection with puromycin (1 $\mathrm{\mu g} /$ $\mathrm{mL}$; Sigma). Flp-mediated deletion was verified by PCR and Southern blot analyses. PCR was performed with the primers P1 (5'-ATCCTCGCCATGGCAACGCGA) and P2 (5'-AGTACCA CAGAATAGAGCACG); wild-type and flox alleles yield fragments of 252 and $361 \mathrm{bp}$, respectively. One clone (F128-10) that was shown to have lost neo was used in the present study. F94 or F128-10 cells were injected into blastocysts of C57BL/ $6 \mathrm{Cr} \times \mathrm{BDF}_{1}$ mice (SLC, Shizuoka Japan), resulting in the birth of chimeric animals. Male chimeras derived from each ES cell line were bred with $\mathrm{C} 57 \mathrm{BL} / 6 \mathrm{Cr}$ females, yielding heterozygous $\mathrm{F}_{1}$ offspring (C57BL/6Cr $\times 129$ background). To generate a null allele $\left(F_{O X H 1^{-}}\right)$, we crossed male chimeras with CAG-Cre transgenic mice (Sakai and Miyazaki 1997) to excise the loxP cassette. The resulting $\mathrm{F}_{1}$ offspring were verified by Southern hybridization and PCR analysis with primers $\mathrm{P} 1, \mathrm{P} 2$, and $\mathrm{P} 3$ (5'GACTGGGTGGCTGATAAGGCT); wild-type and excised alleles yield fragments of 252 and $592 \mathrm{bp}$, respectively. The $\mathrm{F}_{1}$ heterozygotes were crossed with each other, producing FoxH1 $1^{\text {neo/neo }}, F_{O X H} 1^{\text {flox } / \text { flox }}$, and FoxH1 $1^{-/-}$mice. For RT-PCR analysis, total RNA was prepared from E7.75 embryos with guanidine isocianate, and was reverse transcribed with oligo (dT). For detecting FoxH1 mRNA, cDNA was subjected to PCR with the primers $5^{\prime}$-ATCCGTCAGGTCCAGGCAGTG-3' and 5'CTTGGCGAAAGCTCTGTG-3'. To detect Hprt mRNA as a control, the same cDNA was amplified with the primers $5^{\prime}$ AGCGATGATGAACCAGGTTA-3' and 5'-GTTGAGAGAT CATCTCCACC-3'.

\section{In situ hybridization and histology}

Mouse embryos were staged on the basis of their morphology (Downs and Davies 1993). Whole-mount in situ hybridization was performed according to standard procedures (Wilkinson 1992). Wild-type and mutant embryos were processed in the same tube. Embryos were genotyped by PCR analysis of yolk sac DNA with primers P1 and P2 for the wild-type FoxH1 allele, and with primers P1 and P3 for the FoxH1 null allele. For histology, embryos were fixed with Bouin's solution, embedded in paraffin, and sectioned at a thickness of $8 \mu \mathrm{m}$. Sections were stained with hematoxylin and eosin.

\section{Analysis of genetic interaction between FoxH1 and nodal}

Mice that contain an IRES-lacZ cassette in the second exon of nodal have been described previously (Collignon et al. 1996). We crossed nodal ${ }^{\text {lac } /+}$ mice with FoxH1 $1^{+/-}$mice to obtain 
double heterozygotes. Embryos obtained by intercrossing of the double heterozygotes or by crossing the double heterozygotes with $\mathrm{FOxH1}^{+/-}$animals were analyzed. The genotype of each embryo was determined by PCR. The expression of nodal in these embryos was monitored by staining with X-Gal.

\section{Whole-embryo culture}

E7.5 embryos were cultured for $24 \mathrm{~h}$ in $50-\mathrm{mL}$ disposable tubes containing $2 \mathrm{~mL}$ of $50 \%$ Dulbecco's modified Eagle's medium supplemented with $50 \%$ rat serum, as described (Lawson et al. 1986); this volume of medium was sufficient for culturing four embryos (Sturm and Tam 1993). The tubes were filled with a mixture of $5 \% \mathrm{CO}_{2}, 5 \% \mathrm{O}_{2}$, and $90 \% \mathrm{~N}_{2}$ and were rotated at 30 rpm on a roller apparatus in an incubator.

\section{Epiblast-specific deletion of FoxH1}

The lefty2-Cre transgene was constructed by ligating the $5.5-\mathrm{kb}$ upstream region of lefty2 to a Cre cassette derived from pBS-Cre (kindly provided by $\mathrm{H}$. Kondoh). Several mouse lines containing this transgene were established. To examine the specificity of Cre expression, we crossed each line with transgenic mice that harbor a lacZ gene that can be expressed only after Cre-mediated excision. Embryos were genotyped and stained with X-Gal. One transgenic line (21B) that shows epiblast-specific expression of Cre was used in this study. Line 21B animals were crossed with $\mathrm{FOxH}^{+/-}$mice to obtain heterozygotes harboring the Cre transgene; these FoxH1 ${ }^{+/}$,lefty2-Cre mice were mated with FoxH $1^{\text {flox/flox }}$ animals, and the resulting embryos were analyzed at E7.0, E8.5, and E10.5. The genotype of each embryo was determined by PCR analysis with primers P1, P2, and P3; wild-type, null, and flox FoxH1 alleles yield fragments of 252, 592 , and $361 \mathrm{bp}$, respectively. The lefty2-Cre transgene was detected by PCR with a pair of primers specific for Cre as described (Sakai and Miyazaki 1997).

\section{Generation and analysis of chimera embryos}

Chimeras were generated by blastocyst injection as described (Bradley 1987). Blastocysts were collected from intercross between $\mathrm{FOXH}^{+/-}$animals and were injected with wild-type ES (ROSA26) cells at a ratio of 2:14 ES cells/blastocyst. Chimeric embryos were recovered at E8.5, fixed and processed for $\beta$-galactosidase staining with X-Gal (5-bromo-4-chloro-3-indoyl- $\beta$ D-galactoside). The genotype of the host blastocyst was determined retrospectively with extraembryonic tissues. Briefly, individual visceral yolk sacs were dissected from conceptuses, washed in phosphate-buffered saline (PBS), and the endoderm layer were isolated following digestion with pancreatic tyrosine as described (Hogan et al. 1994). DNA samples prepared from the endodermal layer were genotyped with respect to the FoxH1 locus by PCR using primers P1, P2, and P3.

\section{Acknowledgments}

We thank A. Shimono and H. Kondoh for introducing us to ES cell-mediated gene targeting; E.J. Robertson for nodal ${ }^{\text {lacZ }}$ mice; J. Miyazaki for CAG-Cre mice; S. Aizawa, R. Beddington, E. De Robertis, P. Gruss, B. Hermann, B. Hogan, G. Martin, I. Matsuo, Y. Saga, H. Sasaki, and C. Wright for sharing reagents used in this study; and H. Hashiguchi, A. Hirao, S. Ohishi, K. Yamashita, M. Nishijima, Y. Masaoka, K. Miyama, and T. Tanabe for technical assistance. ROSA26 ES cells were provided by S. Aizawa with permissions from P. Soriano and E. Robertson. This work was supported by a grant from CREST (Core Research for Evolutional Science and Technologyl of the Japan Science and Technology Corporation (to H.H.), grants from the Ministry of Education, Science, Sports, and Culture of Japan (to C.M., Y. Saijoh, and H.H.), and a grant from the Uehara Memorial Foundation (to Y. Saijoh).

The publication costs of this article were defrayed in part by payment of page charges. This article must therefore be hereby marked "advertisement" in accordance with 18 USC section 1734 solely to indicate this fact.

\section{References}

Acampora, D., Mazan, S., Lallemand, Y., Avantaggiato, V., Maury, M., Simeone, A., and Brulet, P. 1995. Forebrain and midbrain regions are deleted in $\mathrm{Otx} 2^{-/-}$mutants due to a defective anterior neuroectoderm specification during gastrulation. Development 121: 3279-3290.

Adachi, H., Saijoh, Y., Mochida, K., Ohishi, S., Hashiguchi, H., Hirao, A., and Hamada, H. 1999. Determination of left-right asymmetric expression of nodal by a left side-specific enhancer with sequence similarity to a lefty-2 enhancer. Genes \& Dev. 13: 1589-1600.

Ang, S.-L. and Rossant, J. 1994. HNF3 $\beta$ is essential for node and notochord formation in mouse development. Cell 78: 561574.

Ang, S.-L., Conlon, R.A., Jin, O., and Rossant, J. 1994. Positive and negative signals from mesoderm regulate the expression of mouse Otx2 in ectoderm explants. Development 120: 2979-2989.

Ang, S.-L., Jin, O., Rhinn, M., Daigle, N., Stevenson, L., and Rossant, J. 1998. A targeted mouse Otx2 mutation leads to severe defects in gastrulation and formation of axial mesoderm and to deletion of rostral brain. Development 122: 243-252.

Beddington, R. and Robertson, E.J. 1998. Anterior patterning. Trends Genet. 14: 277-284.

- 1999. Axis development and early asymmetry in mammals. Cell 96: 195-209.

Bradley, A. 1987. Production and analysis of chimeric mice. In Teratocarcinomas and embryonic stem cells: A practical approach (ed. E.J. Robertson), pp. 131-151. IRL Press, Oxford, UK.

Chen, X., Rubock, M.J., and Whitman, M. 1996. A transcriptional partner for Mad proteins in TGF $\beta$ signaling. Nature 383: 691-696.

Chen, X., Weisberg, E., Fridmacher, V., Watanabe, M., Naco, G., and Whitman, M. 1997. Smad4 and FAST-1 in the assembly of activin-response factor. Nature 389: 85-89.

Collignon, J., Varlet, I., and Robertson, E.J. 1996. Relationship between asymmetric nodal expression and the direction of embryonic turning. Nature 381: 155-158.

Conlon, F.L., Lyons, K.M., Takaesu, N., Barth, K.S., Kispert, A.K., Herrmann, B., and Robertson, E.J. 1994. A primary requirement of nodal in the formation and maintenance of the primitive streak in mouse. Development 120: 1919-1928.

Crossley, P.H. and Martin, G.R. 1995. The mouse Fgf8 gene encodes a family of polypeptides and is expressed in regions that direct outgrowth and patterning in the developing embryo. Development 121: 439-451.

Ding, J., Yang, L., Yan, Y.-T., Chen, A., Desai, N., WynshawBoris, A., and Shen, M.M. 1998. Cripto is required for correct orientation of the anterior-posterior axis in the mouse embryo. Nature 395: 702-707. 
Downs, K.M. and Davies, T. 1993. Staging of gastrulating mouse embryos by morphological landmarks in the dissecting microscope. Development 118: 1255-1266.

Gritsman, K., Zhang, J., Cheng, S., Heckscher, E., Talbot, W.S., and Schier, A.F. 1999. The EGF-CFC protein one-eyed pinhead is essential for Nodal signaling. Cell 97: 121-132.

Gu, Z., Nomura, M., Simpson, B.B., Lei, H., Feijen, A., Van den Eijnden-van Raaij, J., Donahoe, P.K., and Li, E. 1998. The type III activin receptor ActRIB is required for egg cylinder organization and gastrulation in the mouse. Genes \& Dev. 12: 844-857.

Harland, R. and Gerhart, J. 1997. Formation and function of Spemann's organizer. Annu. Rev. Cell. Dev. Biol. 13: 611667.

Heyer, J., Escalante-Alcade, D., Lia, M., Boettinger, E., Edelman, W., Stewart, C., and Kucherlapati, R. 1999. Postgastrulation Smad2-deficient embryos show defects in embryo turning and anterior morphogenesis. Proc. Natl. Acad. Sci. 96: $12595-12600$.

Hogan, B., Beddington, R., Costantini, F. and Lacy, E. 1994. Manipulating the mouse embryo: A laboratory manual. Cold Spring Harbor Laboratory Press, Cold Spring Harbor NY.

Hoodless, P., Pye, M., Chaxaud, C., Labbe, E., Attisano, L., Rossant, J., and Wrana, J.L. 2001. Fast functions to specify the anterior primitive streak in the mouse. Gene \& Dev. 15: 1257-1271.

Kimura, C., Yoshinaga, K., Tian, E., Suzuki, M., Aizawa, S., and Matsuo, I. 2000. Visceral endoderm mediates forebrain development by suppressing posteriorizing signals. Dev. Biol. 225: 304-321.

Kispert, A. and Herrmann, B.G. 1994. Immunohistochemical analysis of the Brachyury protein in wild-type IIInd mutant mouse embryos. Dev. Biol. 161: 179-193.

Labbe, E., Silvestri, C., Hoodless, P.A., Wrana, J.L., and Attisano, L. 1998. Smad2 and Smad3 positively and negatively regulate TGF $\beta$-dependent transcription through the forkhead DNA-binding protein FAST2. Mol. Cell 2: 109-120.

Lawson, K.A., Meneses, J.J., and Pedersen, R.A. 1986. Cell fate and lineage in the endoderm of the presomite mouse embryo, studied with an intercellular tracer. Dev. Biol. 115: 325-339.

Liu, B., Dou, C.L., Prabhu, L., and Lai, E. 1999. FAST2 is a mammalian winged-helix protein which mediates TGF $\beta$ signals. Mol. Cell. Biol. 19: 424-430.

Massague, J. 1998. TGF $\beta$ signal transduction. Annu. Rev. Biochem. 67: 753-791.

Matsuo, I., Kuratani, S., Kimura, C., Takeda, N., and Aizawa, S. 1995. Mouse Otx2 functions in the formation and patterning of rostral head. Genes \& Dev. 9: 2646-2658.

Meno, C., Ito, Y., Saijoh, Y., Matsuda, Y., Tashiro, K., and Hamada, H. 1997. Two closely related left-right asymmetrically expressed genes, lefty-1 and lefty-2: Their distinct expression domains, chromosomal linkage and direct neuralizing activity in Xenopus embryos. Genes Cells 2: 513-524.

Meno, C., Gritmann, K., Ohfuji, Y., Heckscher, E., Ohishi, S., Mochida, K., Shimono, A., Kondoh, H., Talbot, W., Robertson, E.J., et al. 1999. Mouse Lefty2 and zebrafish Antivin are feedback inhibitors of Nodal signaling during vertebrate gastrulation. Mol. Cell 4: 287-298.

Meyers, E.N., Lewandoski, M., and Martin, G.R. 1998. An Fgf8 mutant allelic series generated by Cre- and Flp-mediated recombination. Nature Genet. 18: 136-141.

Nomura, M. and Li, E. 1998. Smad2 role in mesoderm formation, left-right patterning and craniofacial development. $\mathrm{Na}$ ture 393: 786-790.

Norris, D.P. and Robertson, E.J. 1999. Node-specific and asym- metric nodal expression patterns are controlled by two distinct cis-acting regulatory elements. Genes \& Dev. 13: $1575-1589$.

Osada, S., Saijoh, Y., Frisch, A., Yeo, C.-Y., Adachi, H., Watanabe, M., Whitman, M., Hamada, H., and Wright, C.V. 2000. Activin/Nodal responsiveness and asymmetric expression of Xenopus nodal-related gene converge on a FAST-regulated module in intron 1. Development 127: 2503-2514.

Piccolo, S., Agius, E., Leyns, L., Bhattacharyya, S., Grunz, H., Boumeester, T., and De Robertis, E.M. 1999. The head inducer Cerberus is a multifunctional antagonist of Nodal, BMP, and Wnt signals. Nature 397: 707-710.

Pogoda, H.-M., Solnica-Krezel, L. Driever, W., and Meyer, D. 2000. The zebrafish forkhead transcription factor FoxH1/ Fast1 is a modulator of Nodal signaling required for organizer formation. Curr. Biol. 10: 1041-1049.

Rhinn, M., Dierich, A., Shawlot, W., Behringer, R.R., Le Meur, M., and Ang, S.L. 1998. Sequential roles for Otx2 in visceral endoderm and neuroectoderm for forebrain and midbrain induction and specification. Development 125: 845-856.

Saijoh, Y., Adachi, H., Mochida, K., Ohishi, S., Hirao, A., and Hamada, H. 1999. Distinct transcriptional regulatory mechanisms underlie left-right asymmetric expression of lefty-1 and lefty-2. Genes \& Dev. 13: 259-269.

Saijoh, Y., Adachi, H., Sakuma, R., Yeo, C.-Y., Yashiro, K., Watanabe, M., Hashiguchi, H., Yashiro, K., Kawabata, M., Miyazono, K., et al. 2000. Left-right asymmetric expression of lefty2 and nodal is induced by a signaling pathway that includes a transcription factor FAST2. Mol. Cell 5: 35-47.

Sakai, K. and Miyazaki, J. 1997. A transgenic mouse line that retains Cre recombinase activity in mature oocytes irrespective of the Cre transgene transmission. Biochem. Biophys. Res. Commun. 237: 318-324.

Sasaki, H. and Hogan, B.L.M. 1993. Differential expression of multiple fork head related genes during gastrulation and axial pattern formation in the mouse embryo. Development 118: 47-59.

Sawai, S., Shimono, A., Hanaoka, K., and Kondoh, H. 1991. Embryonic lethality resulting from disruption of both $N$-myc alleles in mouse zygotes. New Biologist 9: 861-869.

Schier, A.F. and Shen, M.M. 2000. Nodal signaling in vertebrate development. Nature 403: 385-389.

Shawlot, W. and Behringer, R.R. 1995. Requirement for Lim1 in head organizer function. Nature 374: 425-430.

Shawlot, W., Wakamiya, M., Kwan, K.M., Kania, A., Jessel, T., and Behringer, R.R. 1999. Lim1 is required in both primitive streak-derived tissues and visceral endoderm for head formation in the mouse. Development 126: 4925-4932.

Sirotkin, H.I., Gates, M.A., Kelley, P.D., Schier, A.F., and Talbot, W.S. 2000. Fast1 is required for the development of dorsal axial structures in zebrafish. Curr. Biol. 10: 1051-1054.

Song, J., Oh, S.P., Schrewe, H., Nomura, M., Lei, H., Okano, H., Gridley, T., and Li, E. 1999. The type II activin receptors are essential for egg cylinder growth, gastrulation, and rostral head development in mice. Dev. Biol. 213: 157-169.

Sturm, K. and Tam, P.P.L. 1993. Isolation and culture of whole postimplantation embryos and germ layer derivatives. In Guide to techniques in mouse development (eds. P.M. Wassarman and M.L. DePamphilis), pp. 164-190. Academic Press, San Diego, CA.

Tam, P.P.L. and Beddington, R.S.P. 1987. The formation of mesodermal tissues in the mouse embryo during gastrulation and early organogenesis. Development 99: 109-126.

Tam, P.P.L. and Steiner, K.A. 1999. Anterior patterning by synergistic interaction of the early gastrula organizer and the anterior germ layer tissues of the mouse embryo. Develop- 
ment 126: 5171-5179.

Taniguchi, M., Yuasa, S., Fujisawa, H., Naruse, I., Saga, S., Mishima, M., and Yagi, T. 1997. Disruption of Semaphorin $I I I / D$ gene causes severe abnormality in peripheral nerve projection. Neuron 19: 519-530.

Thomas, P. and Beddington, R. 1996 Anterior primitive endoderm may be responsible for patterning the anterior neural plate in the mouse embryo. Curr. Biol. 6: 1487-1496.

Thomas, P.Q., Brown, A., and Beddington, R.S. 1998. Hex: A homeobox gene revealing peri-implantation asymmetry in the mouse embryo and an early transient marker of endothelial cell precursors. Development 125: 85-94.

Toyama, R., O'Connell, M.L., Wright, C.V., Kuehn, M.R., and Dawid, I.B. 1995. Nodal induces ectopic goosecoid and lim1 expression and axis duplication in zebrafish. Development 121: 383-391.

Tremblay, K.D., Hoodless, P.A., Bikoff, E.K., and Robertson, E.J. 2000. Formation of the definitive endoderm in mouse is a Smad2-dependent process. Development 127: 3079-3090.

Varlet, I., Collignon, J., and Robertson, E.J. 1997. nodal expression in the primitive endoderm is required for the specification of the anterior axis during mouse gastrulation. Development 124: 1033-1044.

Waldrip, W.R., Bikoff, E.K., Hoodless, P.A., Wrana, J.L., and Robertson, E.J. 1998. Smad2 signaling in extraembryonic tissues determines anterior-posterior polarity of the early mouse embryo. Cell 92: 797-808.

Watanabe, M. and Whitman, M. 1999. FAST-1 is a key maternal effector of mesoderm inducers in the early Xenopus embryo. Development 126: 5621-5634.

Weinstein, D.C., Ruiz i Altaba, A., Chen, W.S., Hoodless, P., Prezioso, V.R., Jessel, T.M., and Darnell, J.E., Jr. 1994. The winged-helix transcription factor HNF3 $\beta$ is required for notochord development in the mouse embryo. Cell 78: 575588 .

Weisberg, E., Winner, G.E., Chen, X., Farnsworth, C., Hogan, B.L.M., and Whitman, M. 1998. A mouse homologue of FAST-1 transduces TGF $\beta$ superfamily signals and is expressed during early embryogenesis. Mech. Dev. 79: 17-27.

Whitman, M. 1998. Smads and early developmental signaling by the TGFß superfamily. Genes \& Dev. 12: 2445-2462.

Wilkinson, D.G. 1992. Whole mount in situ hybridization of vertebrate embryos. In In situ hybridization: a practical approach (ed. D.G. Wilkinson), pp. 75-84. IRL Press, Oxford, UK.

Zhou, S., Zawel, L., Lengauer, C., Kinzler, K.W., and Vogelstein, B. 1998. Characterization of human FAST-1, a TGFß and activin signal transducer. Mol. Cell 2: 121-127.

Zhou, X., Sasaki, H., Lowe, L., Hogan, B.L., and Kuehn, M.R. 1993. Nodal is a novel TGF- $\beta-$ like gene expressed in the mouse node during gastrulation. Nature 361: 543-547. 


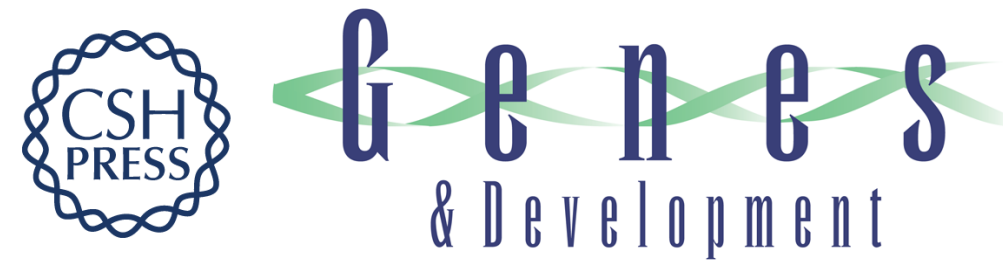

\section{The transcription factor FoxH1 (FAST) mediates Nodal signaling during anterior-posterior patterning and node formation in the mouse}

Masamichi Yamamoto, Chikara Meno, Yasuo Sakai, et al.

Genes Dev. 2001, 15:

Access the most recent version at doi:10.1101/gad.883901

References

This article cites 60 articles, 26 of which can be accessed free at:

http://genesdev.cshlp.org/content/15/10/1242.full.html\#ref-list-1

License

Email Alerting

Receive free email alerts when new articles cite this article - sign up in the box at the top

Service right corner of the article or click here.

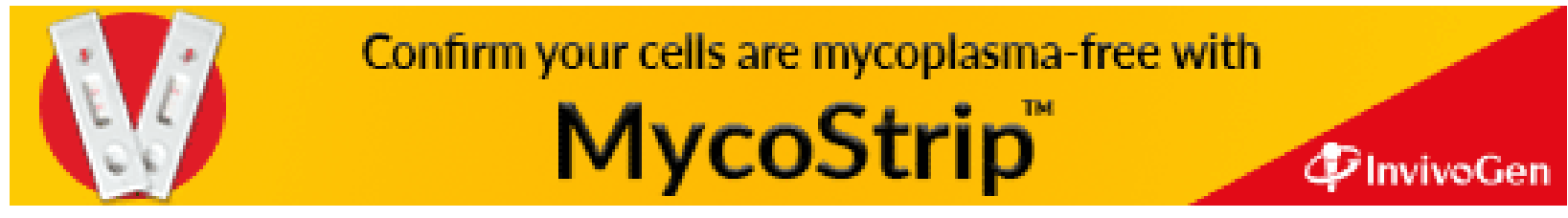

